\title{
Dynamics of Shadow System of a Singular Gierer-Meinhardt System on an Evolving Domain
}

\author{
Nikos I. Kavallaris ${ }^{1}$ (D) Raquel Barreira ${ }^{2,3} \cdot$ Anotida Madzvamuse $^{4,5,6}$ (D) \\ Received: 28 February 2020 / Accepted: 23 October 2020 / Published online: 18 December 2020 \\ (c) The Author(s) 2020
}

\begin{abstract}
The main purpose of the current paper is to contribute towards the comprehension of the dynamics of the shadow system of a singular Gierer-Meinhardt model on an isotropically evolving domain. In the case where the inhibitor's response to the activator's growth is rather weak, then the shadow system of the Gierer-Meinhardt model is reduced to a single though non-local equation whose dynamics is thoroughly investigated throughout the manuscript. The main focus is on the derivation of blow-up results for this non-local equation, which can be interpreted as instability patterns of the shadow system. In particular, a diffusion-driven instability (DDI), or Turing instability, in the neighbourhood of a constant stationary solution, which then is destabilised via diffusion-driven blow-up, is observed. The latter indicates the formation of some unstable patterns, whilst some stability results of global-in-time solutions towards non-constant steady states guarantee the occurrence of some stable patterns. Most of the theoretical results are verified numerically, whilst the numerical approach is also used to exhibit the dynamics of the shadow system when analytical methods fail.
\end{abstract}

Keywords Pattern formation · Turing instability · Activator-inhibitor system · Shadow-system · Invariant regions · Diffusion-driven blow-up · Evolving domains

Mathematics Subject Classification Primary: 35B44 · 35K51; Secondary: 35B36 . 92Bxx

\section{Introduction}

The purpose of the current work is to study an activator-inhibitor system, introduced by Gierer and Meinhardt (1972) to describe the phenomenon of morphogenesis in hydra,

Dedicated to Professor Ioannis Stratis on the occasion of his 65th birthday.

Communicated by Michael Ward.

Extended author information available on the last page of the article 
on an isotropically evolving domain. In particular, a singular Gierer-Meinhardt system on a stationary domain $\Omega \subset \mathbb{R}^{N}, N \geq 1$ with smooth boundary is given by (Gierer and Meinhardt 1972),

$$
\begin{aligned}
u_{t} & =D_{1} \Delta u-u+\frac{u^{p}}{v^{q}}, \quad x \in \Omega, \quad t \in(0, T), \\
\tau v_{t} & =D_{2} \Delta v-v+\frac{u^{r}}{v^{s}}, \quad x \in \Omega, \quad t \in(0, T), \\
\frac{\partial u}{\partial v} & =\frac{\partial v}{\partial v}=0 \quad x \in \partial \Omega_{t}, \quad t \in(0, T), \\
u(x, 0) & =u_{0}(x)>0, \quad v(x, 0)=v_{0}(x)>0, \quad x \in \Omega_{0} \subset \mathbb{R}^{N},
\end{aligned}
$$

where $u(x, t)$ stands for the concentration of the activator, at a spatial point $x \in \Omega$ at time $t \in[0, T], T>0$, which enhances its own production and that of the inhibitor whose concentration is denoted by $v(x, t)$ according to (1.1). On the other hand, the presence of the inhibitor suppresses its own production as well as that of the activator as it is described by (1.2). Also $v$ denotes the unit normal vector on $\partial \Omega$.

Here, $D_{1}$ and $D_{2}$ are the diffusion coefficients of the activator and inhibitor, respectively; $\tau$ represents the response of the inhibitor to the activator's growth. Moreover, the exponents satisfying the conditions: $p>1, q, r,>0$, and $s>-1$, measure the interactions between morphogens. The dynamics of system (1.1)-(1.4) is controlled by two values: the net self-activation index $\psi=(p-1) / r$ and the net cross-inhibition index $\gamma=q /(s+1)$. Index $\xi$ correlates the strength of self-activation of the activator with the cross-activation of the inhibitor. Thus, if $\xi$ is large, then the net growth of the activator is large no matter the growth of the inhibitor. The parameter $\gamma$ measures how strongly the inhibitor suppresses the production of the activator and that of itself. If $\gamma$ is large, then the production of the activator is strongly suppressed by the inhibitor. Finally, the parameter $\tau$ quantifies the inhibitor's response against the activator's growth, cf. Gierer and Meinhardt (1972). Guided by biological interpretation as well as by mathematical reasons, we assume that the parameters $p, q, r, s$ satisfy the condition

$$
p-r \gamma<1
$$

which in the literature is known as the Turing condition. Indeed, as it is pointed in the seminal paper (Gierer and Meinhardt 1972), condition (1.5) guarantees the occurrence of patterns, induced by diffusion, for the solutions of system (1.1)-(1.4), see also Ni et al. (2006) and Ni (2011).

Apart from its biological importance, system (1.1)-(1.4) has a very rich mathematical structure including emerging singularities and thus its dynamics has been extensively study the last few years. More precisely, a thorough study of the structure of its stationary solutions is given in Ni et al. (2006), whilst some global-in-time existence results were proven in Jiang (2006), Li et al. (1995), Masuda and Takahashi (1987) and Rothe (1984) among others. The author in Jiang (2006) proved that under the condition $\psi=\frac{p-1}{r}<1$, a global-in-time solution exists, which is an almost 
optimal result, also taking into consideration the results in Ni et al. (2006). Moreover, in Karali et al. (2013) one can find an investigation of the asymptotic behaviour of the solution of (1.1)-(1.4). The occurrence of finite-time blow-up, which actually implies unlimited growth for the activator, was first established in Li et al. (1995) and later in Karch et al. (2016), Li et al. (2017) and Zou (2015), whereas the case of non-diffusing activator finite-time blow-up was investigated in Karch et al. (2016). The existence and stability of spiky stationary solutions was thoroughly studied in the survey paper (Wei 2008).

Now, in the case that the domain of the interaction of activator and inhibitor, denoted by $\Omega_{t}$, is evolving in time, then the dynamics of this interaction can be described by the following reaction-diffusion system

$$
\begin{aligned}
& u_{t}+\nabla \cdot(\vec{\alpha} u)=D_{1} \Delta u-u+\frac{u^{p}}{v^{q}}, \quad x \in \Omega_{t}, \quad t \in(0, T), \\
& \tau v_{t}+\nabla \cdot(\vec{\alpha} v)=D_{2} \Delta v-v+\frac{u^{r}}{v^{s}}, \quad x \in \Omega_{t}, \quad t \in(0, T), \\
& \frac{\partial u}{\partial v}=\frac{\partial v}{\partial v}=0 \quad x \in \partial \Omega_{t}, \quad t \in(0, T), \\
& u(x, 0)=u_{0}(x)>0, \quad v(x, 0)=v_{0}(x)>0, \quad x \in \Omega_{0} \subset \mathbb{R}^{N},
\end{aligned}
$$

where $\vec{\alpha} \in \mathbb{R}^{N}$ stands for the convection velocity, induced by the material deformation due to the evolution of the domain and $\Omega_{0} \subset \mathbb{R}^{N}$ is the initial domain profile which has smooth boundary $\partial \Omega_{0}$. The initial datum $u_{0}, v_{0}$ are considered bounded, i.e.

$$
u_{0}, v_{0} \in L^{\infty}\left(\Omega_{0}\right)
$$

In the current work, we will only consider the case of an isotropic flow on an evolving domain, whilst the anisotropic case will be investigated in a forthcoming paper. Thus, for any $x \in \Omega_{t}$ we have:

$$
x=\rho(t) \xi, \text { for } \xi \in \Omega_{0} \subset \mathbb{R}^{N},
$$

where $\Omega_{0}$ is an open and bounded $C^{1}-$ domain of $\mathbb{R}^{N}$. Uniform isotropic growth is a plausible biological assumption whereby the domain is assumed to expand uniformly at the same rate in all directions at all times. Examples illustrating isotropically evolving biological surfaces include the famous Nature paper by Kondo and Asai (1995) that depicted mode doubling in pigmentation patterns of the angelfish Pomacanthus as it grows from juvenile to adulthood.

To proceed, we take $\rho(t)$ to be a $C^{1}$-function with $\rho(0)=1$. In the case of a growing domain, we have $\dot{\rho}(t)=\frac{\mathrm{d} \rho}{\mathrm{d} t}>0$, whilst when the domain shrinks or for domain contraction $\dot{\rho}(t)=\frac{\mathrm{d} \rho}{\mathrm{d} t}<0$. Furthermore, the following equality holds

$$
\frac{\mathrm{d} x}{\mathrm{~d} t}=\vec{\alpha}(x, t)
$$


Setting $\hat{u}(\xi, t)=u(\rho(t) \xi, t), \hat{v}(\xi, t)=v(\rho(t) \xi, t)$, and then using the chain rule as well as (1.11) and (1.12), see also Madzvamuse and Maini (2007), we obtain:

$$
\begin{aligned}
& \hat{u}_{t}-\vec{\alpha} \cdot \nabla_{x} u=u_{t}, \quad \nabla_{x} u=\frac{1}{\rho(t)} \nabla_{\xi} \hat{u} \\
& \Delta_{x} u=\frac{1}{\rho^{2}(t)} \Delta_{\xi} \hat{u}, \quad \nabla_{x} \cdot(\vec{\alpha} u)=\vec{\alpha} \cdot \nabla_{x} u+N u \frac{\dot{\rho}(t)}{\rho(t)},
\end{aligned}
$$

whilst similar relations hold for $v$ as well. Therefore, (1.6)-(1.9) is reduced to the following reaction-diffusion system on a reference stationary domain $\Omega_{0}$

$$
\begin{aligned}
\hat{u}_{t} & =\frac{D_{1}}{\rho^{2}(t)} \Delta_{\xi} \hat{u}-\left(1+N \frac{\dot{\rho}(t)}{\rho(t)}\right) \hat{u}+\frac{\hat{u}^{p}}{\hat{v}^{q}}, \quad \xi \in \Omega_{0}, \quad t \in(0, T),(1.13) \\
\tau \hat{v}_{t} & =\frac{D_{2}}{\rho^{2}(t)} \Delta_{\xi} \hat{v}-\left(1+N \frac{\dot{\rho}(t)}{\rho(t)}\right) \hat{v}+\frac{\hat{u}^{r}}{\hat{v}^{s}}, \quad \xi \in \Omega_{0}, \quad t \in(0, T),(1.14) \\
\frac{\partial \hat{u}}{\partial v} & =\frac{\partial \hat{v}}{\partial v}=0 \quad \xi \in \partial \Omega_{0}, \quad t \in(0, T), \\
\hat{u}(\xi, 0) & =\hat{u}_{0}(\xi)>0, \quad \hat{v}(\xi, 0)=\hat{v}_{0}(\xi)>0, \quad \xi \in \Omega_{0},
\end{aligned}
$$

where $\Delta_{\xi}$ represents the Laplacian on the reference static domain $\Omega_{0}$. Henceforth, without any loss of generality we will omit the index $\xi$ from the Laplacian.

Defining a new time scale (Labadie (2008)),

$$
\sigma(t)=\int_{0}^{t} \frac{1}{\rho^{2}(\theta)} \mathrm{d} \theta,
$$

and setting $\tilde{u}(\xi, \sigma)=\hat{u}(\xi, t), \tilde{v}(\xi, \sigma)=\hat{v}(\xi, t)$, then system (1.13)-(1.16) can be written as

$$
\begin{aligned}
\tilde{u}_{\sigma}= & D_{1} \Delta_{\xi} \tilde{u} \\
& -\left(\phi^{2}(\sigma)+N \frac{\dot{\phi}(\sigma)}{\phi(\sigma)}\right) \tilde{u}+\phi^{2}(\sigma) \frac{\tilde{u}^{p}}{\tilde{v}^{q}}, \quad \xi \in \Omega_{0}, \sigma \in(0, \Sigma), \\
\tau \tilde{v}_{\sigma}= & D_{2} \Delta_{\xi} \tilde{v} \\
& -\left(\phi^{2}(\sigma)+N \frac{\dot{\phi}(\sigma)}{\phi(\sigma)}\right) \tilde{v}+\phi^{2}(\sigma) \frac{\tilde{u}^{r}}{\tilde{v}^{s}}, \quad \xi \in \Omega_{0}, \sigma \in(0, \Sigma), \quad \xi \in \partial \Omega_{0}, \quad \sigma \in(0, \Sigma), \\
\frac{\partial \tilde{u}}{\partial v}= & \frac{\partial \tilde{v}}{\partial v}=0, \quad \tilde{v}(\xi, 0)=\hat{v}_{0}(\xi)>0, \quad \xi \in \Omega_{0} \\
\tilde{u}(\xi, 0)= & \hat{u}_{0}(\xi)>0, \quad \tilde{v}(\xi)
\end{aligned}
$$

where $\rho(t)=\phi(\sigma)$, and thus $\dot{\rho}(t)=\frac{\dot{\phi}(\sigma)}{\phi^{2}(\sigma)}$, and $\Sigma=\sigma(T)$.

Typically, in cellular biology, molecular species resident in the cytosol are known to diffuse a lot faster than those molecular species resident in the cell membrane [see Cusseddu et al. (2019)] and references therein). Hence, if we assume $D_{1} \ll D_{2}$, where 
the inhibitor diffuses much faster than the activator, then system (1.18)-(1.21) can be fairly approximated by an ODE-PDE system with a non-local reaction term. We will denote the new approximation by shadow system as coined in Keener (1978). Below, we provide a rather rough derivation of the shadow system, while for a more rigorous approach one can appeal to the arguments in Bobrowski and Kunze (2019). Indeed, dividing (1.19) by $D_{2}$ and taking $D_{2} \rightarrow+\infty$, see also Ni (2011), then it follows that $\tilde{v}$ solves

$$
\begin{aligned}
& \Delta_{\xi} \tilde{v}=0, \quad \xi \in \Omega_{0}, \\
& \frac{\partial \tilde{v}}{\partial v}=0, \quad \xi \in \partial \Omega_{0},
\end{aligned}
$$

for any fixed $\sigma \in(0, \Sigma)$. Due to the imposed Neumann boundary condition then $\tilde{v}$ is a spatial homogeneous (independent of $\xi$ ) solution, and thus averaging (1.19) over $\Omega_{0}$ we get that $f_{\Omega_{0}} \tilde{v}(\xi, \sigma) d \xi:=\frac{1}{\left|\Omega_{0}\right|} \int_{\Omega_{0}} \tilde{v}(\xi, \sigma) \mathrm{d} \xi:=\eta(\sigma)$. It follows then that $\eta(\sigma)$ satisfies the following partial differential equation

$$
\tau \frac{\mathrm{d} \eta}{\mathrm{d} \sigma}=-\Phi(\sigma) \eta+\phi^{2}(\sigma) \frac{f_{\Omega_{0}} \tilde{u}^{r}}{\eta^{s}}, \quad \sigma \in(0, \Sigma)
$$

where

$$
\Phi(\sigma)=:\left(\phi^{2}(\sigma)+N \frac{\dot{\phi}(\sigma)}{\phi(\sigma)}\right)
$$

and $f_{\Omega_{0}} \tilde{u}^{r} d \xi:=\frac{1}{\left|\Omega_{0}\right|} \int_{\Omega_{0}} \tilde{u}^{r} \mathrm{~d} \xi$.

Finally, we can infer that the pair $(\tilde{u}, \eta)$ satisfies the shadow system

$$
\begin{aligned}
& \tilde{u}_{\sigma}=D_{1} \Delta_{\xi} \tilde{u}-\Phi(\sigma) \tilde{u}+\phi^{2}(\sigma) \frac{\tilde{u}^{p}}{\eta^{q}}, \quad \xi \in \Omega_{0}, \quad \sigma \in(0, \Sigma), \\
& \tau \frac{d \eta}{d \sigma}=-\Phi(\sigma) \eta+\phi^{2}(\sigma) \frac{f_{\Omega_{0}} \tilde{u}^{r} d \xi}{\eta^{s}}, \quad \sigma \in(0, \Sigma), \\
& \frac{\partial \tilde{u}}{\partial v}=0, \quad \xi \in \partial \Omega_{0}, \quad \sigma \in(0, \Sigma), \\
& \tilde{u}(\xi, 0)=\hat{u}_{0}(\xi)>0, \quad \eta(0)=\eta_{0}:=f_{\Omega_{0}} \tilde{v}(\xi, 0) d \xi>0, \quad \xi \in \Omega_{0} .
\end{aligned}
$$

In the limit case $\tau \rightarrow 0$, i.e. when the inhibitor's response to the growth of the activator is quite small, then the shadow system is reduced to a single, though, nonlocal equation. Indeed, when $\tau=0,(1.25)$ entails that $\eta(\sigma)=\left(\frac{\phi^{2}(\sigma)}{\Phi(\sigma)} f_{\Omega_{0}} \tilde{u}^{r} d \xi\right)^{\frac{1}{s+1}}$, and thus (1.24) $-(1.27)$ reduce to

$$
\tilde{u}_{\sigma}=D_{1} \Delta_{\xi} \tilde{u}-\Phi(\sigma) \tilde{u}+\frac{\Psi(\sigma) \tilde{u}^{p}}{\left(f_{\Omega_{0}} \tilde{u}^{r} d \xi\right)^{\gamma}}, \quad \xi \in \Omega_{0}, \quad \sigma \in(0, \Sigma),
$$




$$
\begin{aligned}
& \frac{\partial \tilde{u}}{\partial v}=0, \quad \xi \in \partial \Omega_{0}, \quad \sigma \in(0, \Sigma), \\
& \tilde{u}(\xi, 0)=\hat{u}_{0}(\xi)>0, \quad \xi \in \Omega_{0},
\end{aligned}
$$

recalling $\gamma=\frac{q}{s+1}$ and

$$
\Psi(\sigma)=\phi^{2(1-\gamma)}(\sigma) \Phi^{\gamma}(\sigma)
$$

Recovering the $t$ variable entails that the following partial differential equation holds

$$
\begin{aligned}
& \hat{u}_{t}=\frac{D_{1}}{\rho^{2}(t)} \Delta_{\xi} \hat{u}-L(t) \hat{u}+L^{-\gamma}(t) \frac{\hat{u}^{p}}{\left(f_{\Omega_{0}} \hat{u}^{r} \mathrm{~d} \xi\right)^{\gamma}}, \quad \xi \in \Omega_{0}, \quad t \in(0, T), \\
& \frac{\partial \hat{u}}{\partial v}=0, \quad \xi \in \partial \Omega_{0}, \quad t \in(0, T) \\
& \hat{u}(\xi, 0)=\hat{u}_{0}(\xi)>0, \quad \xi \in \Omega_{0}
\end{aligned}
$$

where $L(t):=\left(1+N \frac{\dot{\rho}(t)}{\rho(t)}\right)$. We note that formulation (1.28)-(1.30) is more appropriate for the demonstrated mathematical analysis; however, all of our theoretical results can be directly interpreted in terms of the equivalent formulation (1.32)-(1.34). Besides, formulation (1.32)-(1.34) is more appropriate for our numerical experiments since the calculation of the functions $\Phi(\sigma)$ and $\Psi(\sigma)$ is not always possible.

The primary aim of the current work is to investigate the long-time dynamics of the non-local problem (1.28)-(1.30). Then, it is also examined under which circumstances the dynamics of (1.28)-(1.30) resembles that of the reaction-diffusion system (1.18)(1.21), which is not always the case, as it has been pointed out in Jiang (2006), Karali et al. (2013), Li and $\mathrm{Ni}$ (2009), and Li and Yip (2014). The latter study is performed by using analytical methods, but when these methods fail, then a numerical approach will be implemented. We also use the numerical approach to verify the derived analytical results.

Biologically speaking, we will investigate whether it is necessary to study the dynamics of both reactants or only the study of the activator's dynamics is sufficient. This is done under the assumption that the inhibitor's response to the growth of the activator is quite small and that it also diffuses much faster than the activator. From here onwards, we take $D_{1}=1$, revert to the initial variables $x, u$ instead of $\xi, \widetilde{u}$ and we drop the index $\xi$ from the Laplacian $\Delta$ without any loss of generality. Hence, we will focus our study on the following single nonlocal partial differential equation

$$
\begin{aligned}
& u_{\sigma}=\Delta u-\Phi(\sigma) u+\frac{\Psi(\sigma) u^{p}}{\left(f_{\Omega_{0}} u^{r} d x\right)^{\gamma}}, \quad x \in \Omega_{0}, \quad \sigma \in(0, \Sigma), \\
& \frac{\partial u}{\partial v}=0, \quad x \in \partial \Omega_{0}, \quad \sigma \in(0, \Sigma), \\
& u(x, 0)=u_{0}(x)>0, \quad x \in \Omega_{0} .
\end{aligned}
$$


Hence, the layout of the current work is as follows. Section 2 deals with the derivation of various blow-up results, induced by the non-local reaction term (ODE blow-up results), together with some global-time existence results for problem (1.35)-(1.37). The notion of finite-time blow-up should be understood biologically as an overcrowding of activator's population, which mathematically means that $\lim _{\sigma \rightarrow \Sigma_{b}}\|u(\cdot, \sigma)\|_{\infty}=\infty$. The impact of domain growth on the finite-time blow-up of a reaction-diffusion equation was first discussed in Labadie (2008); however, the novelty of our approach, both demonstrated in Sects. 2 and 3, consists of the fact that we investigate both growing and shrinking domains but for a non-local reactiondiffusion equation whose dynamics are more complicated. Following the approach developed in Kavallaris and Suzuki (2017, 2018), in Sect. 3 we present and prove a Turing instability result associated with (1.35)-(1.37). This Turing instability occurs under the Turing condition (1.5) and is exhibited in the form of a diffusion-driven blow-up $(D D B U)$. It is not the first time that $D D B U$ phenomenon is observed, see for example Fila and Ninomiya (2005), Hu and Yin (1995), Kavallaris and Suzuki (2017, 2018) and Mizoguchi et al. (1998), nevertheless according to our knowledge it is the first time that such a result is proven for non-local reaction-diffusion equations defined on evolving domains. Finally, in Sect. 4 we appeal to various numerical experiments in order to confirm some of the theoretical results presented in Sects. 2 and 3. More importantly, the numerical approach is also used to compare the long-time dynamics of the non-local problem (1.35)-(1.37) with that of the reaction-diffusion system (1.24)-(1.27). It is also applied to study the dynamics of the shadow system (1.35)-(1.37) when analytical methods fail to do so.

\section{ODE Blow-up and Global Existence}

The current section is devoted to the presentation of some ODE blow-up results for problem (1.35)-(1.37), i.e. blow-up results induced by the kinetic (non-local) term in (1.35). Here, by blow-up of the solution $u$ of (1.35)-(1.37) we mean the occurrence of a finite time $\Sigma_{b}$ so that $\lim _{\sigma \rightarrow \Sigma_{b}}\|u(\cdot, \sigma)\|=\infty$. Next, some global-in-time existence results for the solution $u$ of (1.35)-(1.37) are also presented, that is $u$ exists for any $\sigma>0$ and it is bounded. It should be pointed out that local-in-time existence of nonlocal problem (1.35)-(1.37) is easily obtained by using ideas in Quittner and Souplet (2007).

Throughout the manuscript, we use the notation $C$ and $c$ to denote positive constants with big and small values, respectively. Our first observation is that the concentration of the activator cannot become zero in finite time. Indeed, the following proposition holds.

\section{Proposition 2.1 Assume that}

$$
\inf _{(0, \Sigma)} \Psi(\sigma):=m_{\Psi}>0, \quad \inf _{(0, \Sigma)} \Phi(\sigma):=m_{\Phi}>0 \text { and } \sup _{(0, \Sigma)} \Phi(\sigma):=M_{\Phi}<+\infty
$$


then for each $\Sigma>0$ there exists $C_{\Sigma}>0$ such that for the solution $u(x, \sigma)$ of (1.35)(1.37) the following inequality holds

$$
u(x, \sigma) \geq C_{\Sigma} \text { in } \Omega_{0} \times[0, \Sigma)
$$

Proof Owing to the maximum principle and by using (2.1), we derive that $u=$ $u(x, \sigma)>0$. By virtue of the comparison principle, we also deduce that $u(x, \sigma) \geq$ $\tilde{u}(\sigma)$, where $\tilde{u}=\tilde{u}(\sigma)$ is the solution to $\frac{d \tilde{u}}{d \sigma}=-M_{\Phi} \tilde{u}$ in $(0, \Sigma), \quad \tilde{u}(0)=\tilde{u}_{0} \equiv$ $\inf _{\Omega_{0}} u_{0}(x)>0$, and thus (2.2) is satisfied with $C=\tilde{u}_{0} \mathrm{e}^{-M_{\Phi} \Sigma}$.

Remark 2.1 It is easily checked that condition (2.1) is satisfied for any decreasing function $\phi(\sigma)$ satisfying

$$
\phi(\sigma)>\frac{1}{\sqrt{2 N \sigma+1}}, 0<\sigma<\Sigma
$$

since then by virtue of (1.23)

$$
0<\Phi(\sigma)=\left(\phi^{2}(\sigma)+N \frac{\dot{\phi}(\sigma)}{\phi(\sigma)}\right)<\phi^{2}(\sigma)<\phi^{2}(0)=1, \quad 0<\sigma<\Sigma
$$

Then, (2.4) via (1.31) implies that

$$
0<\Psi(\sigma)=(\phi(\sigma))^{2(1-\gamma)} \Phi^{\gamma}(\sigma)<1, \quad \text { for } 0<\gamma<1, \quad 0<\sigma<\Sigma
$$

and

$$
0<\Psi(\sigma)=(\phi(\sigma))^{2(1-\gamma)} \Phi^{\gamma}(\sigma)<m_{\Phi}^{2(1-\gamma)}, \text { for } \gamma>1, \quad 0<\sigma<\Sigma,
$$

when $m_{\Phi}=\inf _{(0, \Sigma)} \Phi(\sigma)>0$.

A key estimate for obtaining some blow-up results presented throughout is the following proposition.

Proposition 2.2 Let $\Psi(\sigma)$ and $\Phi(\sigma)$ satisfy (2.1), then there exists $\delta_{0}>0$ such for any $0<\delta \leq \delta_{0}$ the following estimate is fulfilled

$$
\int_{\Omega_{0}} u^{-\delta} \leq C \text { for any } 0<\sigma<\Sigma
$$

where the positive constant $C$ is independent of time $\sigma$.

Proof Define $\chi=u^{\frac{1}{\alpha}}$ for $\alpha \neq 0$, then we can easily check that $\chi$ satisfies

$$
\alpha \chi_{\sigma}=\alpha\left(\Delta \chi+4(\alpha-1)\left|\nabla \chi^{\frac{1}{2}}\right|^{2}\right)
$$




$$
\begin{gathered}
-\Phi \chi+\frac{\Psi u^{p-1+\frac{1}{\alpha}}}{\left(f_{\Omega_{0}} u^{r}\right)^{\gamma}} \text { in } \Omega_{0} \times(0, \Sigma), \\
\frac{\partial \chi}{\partial v}=0, \quad \text { on } \partial \Omega_{0} \times(0, \Sigma), \\
\chi(x, 0)=u_{0}^{\frac{1}{\alpha}}(x), \text { in } \Omega_{0} .
\end{gathered}
$$

Averaging (2.8) over $\Omega_{0}$, we obtain

$$
\alpha \frac{d}{d \sigma} f_{\Omega_{0}} \chi+4 \alpha(1-\alpha) f_{\Omega_{0}}\left|\nabla \chi^{\frac{1}{2}}\right|^{2}+\Phi f_{\Omega_{0}} \chi=\frac{f_{\Omega_{0}} \Psi u^{p-1+\frac{1}{\alpha}}}{\left(f_{\Omega_{0}} u^{r}\right)^{\gamma}}
$$

and hence

$$
\frac{d}{d \sigma} \int_{\Omega_{0}} \chi+4(1-\alpha) f_{\Omega_{0}}\left|\nabla \chi^{\frac{1}{2}}\right|^{2}+\frac{\Phi}{\alpha} f_{\Omega_{0}} \chi \leq 0
$$

for $\alpha<0$. Setting $\delta=-\frac{1}{\alpha}$, we have

$$
\frac{\mathrm{d}}{\mathrm{d} \sigma} f_{\Omega_{0}} \chi+4\left(1+\delta^{-1}\right) f_{\Omega_{0}}\left|\nabla \chi^{\frac{1}{2}}\right|^{2} \leq M_{\Phi} \delta f_{\Omega_{0}} \chi .
$$

Now, recall the Sobolev's inequality, Brezis (2011), that reads

$$
\|\nabla w\|_{2}^{2} \geq C_{1}\left(N, \Omega_{0}\right)\|w\|_{2}^{2}, \quad \text { for any } \quad w \in H^{1}\left(\Omega_{0}\right) \quad \text { and } \quad N \geq 2
$$

where $C_{1}\left(N, \Omega_{0}\right)$ is a positive constant depending only on dimension $N$ and domain $\Omega_{0}$.

Then, by choosing $0<\delta \ll 1$, (2.12) in conjunction with (2.13) and for $w=\chi^{\frac{1}{2}}$ it follows that $\frac{d}{d \sigma} f_{\Omega_{0}} \chi+C_{2} f_{\Omega_{0}} \chi \leq 0$, for some positive constant $C_{2}$. Consequently, Gröwnwall's lemma yields that $\chi(\sigma) \leq C<\infty$ for any $0<\sigma<\Sigma$ and thus (2.7) follows due to the fact that $\chi=u^{-\delta}$.

Remark 2.2 Note that Proposition 2.2 guarantees that the non-local term of problem (1.35)-(1.37) stays away from zero and hence solution $u$ can never decay to zero. In fact, inequality (2.7) implies $f_{\Omega_{0}} u^{\delta} \geq c=C^{-1}$ and then

$$
f_{\Omega_{0}} u^{r} \geq\left(f_{\Omega_{0}} u^{\delta}\right)^{r / \delta} \geq c^{r / \delta}>0 \text { for any } 0<\sigma<\Sigma,
$$

follows by Jensen's inequality, Evans (2010), and taking $\delta \leq r$, where again $c$ is independent of time $\sigma$. The latter estimate rules out the possibility of (finite time or infinite time) quenching, i.e. $\lim _{\sigma \rightarrow \Sigma}\|u(\cdot, \sigma)\|_{\infty}=0$ for $\Sigma<\infty$ or $\Sigma=\infty$, cannot happen, and thus activator's extinction in the long run is not possible. 
Remark 2.3 In case $\Phi(\sigma)$ is not bounded from above, as it happens for $\rho(t)=\mathrm{e}^{\beta t}, \beta>$ 0 , when $\Phi(\sigma)=(1+N \beta)(1-2 \beta \sigma)^{-1}, 0<\sigma<\frac{1}{2 \beta}$, then both of the estimates (2.7) and (2.14) still hold true; however, the involved constants depend on time $\sigma$ and thus (finite or infinite time) quenching cannot be ruled out.

Next, we present our first ODE-type blow-up result for problem (1.35)-(1.37) when an anti-Turing condition, the reverse of (1.5) is satisfied.

Theorem 2.1 Take $p \geq r, 0<\gamma<1$ and $\omega=p-r \gamma>1$. Assume also $\Psi(\sigma)>0$ and consider initial data $u_{0}(x)$ such that

$$
\bar{u}_{0}:=\int_{\Omega_{0}} u_{0} d x>(\omega-1)^{\frac{1}{1-\omega}} I^{\frac{1}{1-\omega}}(\Sigma)>0
$$

provided that

$$
I(\Sigma):=\int_{0}^{\Sigma} \Psi(\theta) \mathrm{e}^{(1-\omega) \int^{\theta} \Phi(\eta) \mathrm{d} \eta} \mathrm{d} \theta<\infty
$$

then the solution of (1.35)-(1.37) blows up in finite time $\Sigma_{b}<\Sigma$, i.e. $\lim _{\sigma \rightarrow \Sigma_{b}}$ $\|u(\cdot, \sigma)\|_{\infty}=+\infty$.

Proof Since $p>1$ and $p \geq r$, then by virtue of the Hölder's inequality $f_{\Omega_{0}} u^{p} \geq$ $\left(f_{\Omega_{0}} u\right)^{p}$ and $\left(f_{\Omega_{0}} u^{r}\right)^{\gamma} \leq\left(f_{\Omega_{0}} u^{p}\right)^{\frac{\gamma r}{p}}$. Then, $\bar{u}(\sigma)=f_{\Omega_{0}} u(x, \sigma) d x$ satisfies

$$
\frac{\mathrm{d} \bar{u}}{\mathrm{~d} \sigma}=-\Phi(\sigma) \bar{u}+\Psi(\sigma) \frac{f_{\Omega_{0}} u^{p}}{\left(f_{\Omega_{0}} u^{r}\right)^{\gamma}} \geq-\Phi(\sigma) \bar{u}+\Psi(\sigma) \bar{u}^{p-r \gamma} \text { for } 0<\sigma<\Sigma .
$$

Set now $F(\sigma)$ to be the solution of the following Bernoulli's type initial value problem $\frac{d F}{d \sigma}=-\Phi(\sigma) F(\sigma)+\Psi(\sigma) F^{\omega}(\sigma), 0<\sigma<\Sigma, \quad F(0)=\bar{u}_{0}>0$, then via the comparison principle $F(\sigma) \leq \bar{u}(\sigma)$ for $0<\sigma<\Sigma$ and $F(\sigma)$ is given by $F(\sigma)=\mathrm{e}^{(\omega-1) \int^{\sigma} \Phi(\eta) d \eta}(G(\sigma))^{\frac{1}{1-\omega}}$, where $G(\sigma):=\left[\bar{u}_{0}^{1-\omega}-(\omega-1)\right.$ $\left.\int_{0}^{\sigma} \Psi(\theta) \mathrm{e}^{(1-\omega) \int^{\theta} \Phi(\eta) \mathrm{d} \eta} \mathrm{d} \theta\right]$. Note that $F(\sigma)$ blows up in finite-time if there exists $\sigma^{*}<\Sigma$ such that $G\left(\sigma^{*}\right)=0$. First note that $G(0)>0$; furthermore, under the assumption (2.15) we have $\lim _{\sigma \rightarrow \Sigma} G(\sigma)<0$ and thus by virtue of the intermediate value theorem there exists $\sigma^{*}<\Sigma$ such that $G\left(\sigma^{*}\right)=0$. The latter implies that $\lim _{\sigma \rightarrow \sigma^{*}} F(\sigma)=+\infty$ and therefore, $\lim _{s \rightarrow \Sigma_{b}} \bar{u}(\sigma)=+\infty$ for some $\Sigma_{b} \leq \sigma^{*}$, which completes the proof.

Remark 2.4 Note that for an exponentially growing domain, i.e. when $\rho(t)=\mathrm{e}^{\beta t}, \beta>$ 0 , condition (2.16) is satisfied since then $1<\Phi(\sigma)=(1+N \beta)(1-2 \beta \sigma)^{-1}$ and $1<\Psi(\sigma)=(1+N \beta)^{\gamma}(1-2 \beta \sigma)^{-1}$ for all $\sigma \in\left(0, \frac{1}{2 \beta}\right)$. Thus,

$$
I(\Sigma)=(1+N \beta)^{\gamma} \int_{0}^{\frac{1}{2 \beta}}(1-2 \beta \theta)^{\frac{(\omega-1)(1+N \beta)}{2 \beta}-1} \mathrm{~d} \theta=\frac{(1+N \beta)^{\gamma-1}}{(\omega-1)}<+\infty
$$


and according to Theorem 2.1 finite-time blow-up takes place at time

$$
\Sigma_{g} \leq \sigma_{g}:=\frac{1}{2 \beta}\left\{1-\left[1-(1+N \beta)^{1-\gamma} \bar{u}_{0}^{1-\omega}\right]^{\frac{2 \beta}{(\omega-1)(1+N \beta)}}\right\},
$$

and for initial data $u_{0}$ satisfying $\bar{u}_{0}>(1+N \beta)^{\frac{1-\gamma}{\omega-1}}$. Notably the bigger the exponent $\beta>0$ is, the faster the evolving domain grows, then a rather large initial condition $u_{0}$ is needed in order to get blow-up according to Theorem 2.1.

Conversely, for an exponentially shrinking domain, i.e. when $\rho(t)=\mathrm{e}^{-\beta t}, 0<$ $\beta<\frac{1}{N}$, then again condition (2.16) is valid since then

$$
0<\Phi(\sigma)=(1-N \beta)(1+2 \beta \sigma)^{-1}<1, \quad \sigma \in(0, \infty)
$$

and

$$
0<\Psi(\sigma)=(1-N \beta)^{\gamma}(1+2 \beta \sigma)^{-1}<1, \quad \sigma \in(0, \infty) .
$$

In that case

$$
I(\Sigma)=(1-N \beta)^{\gamma} \int_{0}^{+\infty}(1+2 \beta \theta)^{\frac{(\omega-1)(1-N \beta)}{2 \beta}-1} \mathrm{~d} \theta=\frac{(1-N \beta)^{\gamma-1}}{(\omega-1)}<+\infty,
$$

and again finite-time blow-up occurs at

$$
\Sigma_{d} \leq \sigma_{d}:=\frac{1}{2 \beta}\left\{\left[1-(1-N \beta)^{1-\gamma} \bar{u}_{0}^{1-\omega}\right]^{\frac{2 \beta}{(1-\omega)(1-N \beta)}}-1\right\} \text {, }
$$

provided that the initial data satisfy $\bar{u}_{0}>(1-N \beta)^{\frac{1-\gamma}{\omega-1}}$. Therefore, the smallest $0<\beta<\frac{1}{N}$ is chosen, the fastest the evolving domain shrinks, then the smaller initial data $u_{0}$ are required for the occurrence of finite-time blow-up predicted by Theorem 2.1 .

For a stationary domain, i.e. when $\rho(t)=\phi(\sigma)=1$, we have $\Phi(\sigma)=\Psi(\sigma)=1$ and thus finite-time blow-up occurs at

$$
\Sigma_{s} \leq \sigma_{s}:=\frac{1}{1-\omega} \ln \left(1-\bar{u}_{0}^{1-\omega}\right)
$$

provided that $\bar{u}_{0}>1$, cf. Kavallaris and Suzuki (2017, 2018).

In conclusion, conditions (2.15) and (2.16) imply, since $\omega>1$ and $\Psi(s)>0$, that the faster the evolving domain expands, then the bigger initial data are required to obtain finite-time blow-up. On the other hand, the faster the evolving domain shrinks, then the smaller initial data $u_{0}$ are needed for finite-time blow-up to occur.

Note also that by relations (2.18), (2.21) and (2.22), we cannot really obtain an ordering of blowing-up times $\Sigma_{g}, \Sigma_{d}$ and $\Sigma_{s}$ since there is not a clear ordering of the 
corresponding upper bounds $\sigma_{g}, \sigma_{d}, \sigma_{s}$ However, we conjecture that $\Sigma_{g}>\Sigma_{s}>\Sigma_{d}$, a conjecture which is verified by numerical Experiment 1 in Sect. 4; see in particular Fig. 1.

Remark 2.5 When the domain evolves logistically, which is a feasible choice in the context of biology, cf. Plaza et al. (2004), i.e. when $\rho(t)=\frac{\mathrm{e}^{\beta t}}{1+\frac{1}{m}\left(\mathrm{e}^{\beta t}-1\right)}$ for $m \neq 1$, then equation (1.17) cannot be solved for $t$ and it is more convenient to deal with problem (1.32)-(1.34) instead. Then, following the same approach as in Theorem 2.1 it can be shown that the solution of (1.32)-(1.34) exhibits finite-time blow-up under the same conditions for parameters $p, \gamma, r$ provided that the initial condition satisfies

$$
\bar{u}_{0}:=\int_{\Omega_{0}} u_{0} \mathrm{~d} x>(\omega-1)^{\frac{1}{1-\omega}} \int_{0}^{\infty} L^{-\gamma}(\theta) \mathrm{e}^{(1-\omega) \int^{\theta} L(\eta) \mathrm{d} \eta} \mathrm{d} \theta,
$$

where now the quantity $L(t)=1+\frac{N \beta\left(1-\frac{1}{m}\right)}{1+\frac{1}{m}\left(\mathrm{e}^{\beta t}-1\right)}$.

Remark 2.6 Assume now that

$$
0<\bar{u}_{0}<(\omega-1)^{\frac{1}{1-\omega}} I^{\frac{1}{1-\omega}}(\Sigma),
$$

then $G(\Sigma)>0$ and since $G(\sigma)$ is strictly decreasing we get that $G(\sigma)>0$ for any $0<\sigma<\Sigma$ which implies that $F(\sigma)$ never blows up. Therefore, since $F(\sigma) \leq \bar{u}(\sigma)$, there is still a possibility that $\bar{u}(\sigma)$ does not blow up either; however, we cannot be sure and it remains to be verified numerically; more precisely see Fig. 2 of Experiment 1 in Sect. 4.

Next, we investigate the dynamics of some $L^{\ell}$-norms $\|u(\cdot, \sigma)\|_{\ell}$, which identify some invariant regions in the phase space. We first define $\zeta(\sigma)=f_{\Omega_{0}} u^{r} d x, y(\sigma)=$ $f_{\Omega_{0}} u^{-p+1+r} d x$ and $w(\sigma)=f_{\Omega_{0}} u^{p-1+r} d x$, then Hölder's inequality implies

$$
w(\sigma) y(\sigma) \geq \zeta^{2}(\sigma), \quad 0 \leq \sigma<\Sigma
$$

Our first result in this direction provides some conditions under which a finite-time blow-up takes place, when an anti-Turing condition is in place and is stated as follows.

Theorem 2.2 Take $0<\gamma<1$ and $r \leq 1<\frac{p-1}{r}$. Assume that $\Phi(\sigma), \Psi(\sigma)$ satisfy (2.1), then if one of the following conditions holds:

(1) $w(0)<\frac{m_{\Psi}}{M_{\Phi}} \zeta(0)^{1-\gamma}$,

(2) $\frac{p-1}{r} \geq 2$ and $w(0)<1$,

then finite-time blow-up occurs.

Proof Set $\chi=u^{\frac{1}{\alpha}}$ with $\alpha \neq 0$, then following the same steps as in Proposition 2.2 we derive 


$$
\begin{aligned}
& \alpha \chi_{\sigma}=\alpha\left(\Delta \chi+4(\alpha-1)\left|\nabla \chi^{\frac{1}{2}}\right|^{2}\right) \\
& \frac{\partial \chi}{\partial v}=0, \quad x \in \partial \Omega_{0}, \sigma \in(0, \Sigma), \\
& \chi(x, 0)=u_{0}^{\frac{1}{\alpha}}(x), \quad x \in \Omega_{0} .
\end{aligned}
$$

Averaging (2.26) over $\Omega_{0}$ and using zero-flux boundary condition (2.27), we obtain

$$
\alpha \frac{\mathrm{d}}{\mathrm{d} \sigma} f_{\Omega_{0}} \chi=-4 \alpha(1-\alpha) f_{\Omega_{0}}\left|\nabla \chi^{\frac{1}{2}}\right|^{2}-\Phi(\sigma) f_{\Omega_{0}} \chi+\Psi(\sigma) \frac{f_{\Omega_{0}} u^{p-1+\frac{1}{\alpha}}}{\left(f_{\Omega_{0}} u^{r}\right)^{\gamma}}
$$

Relation (2.29) for $\alpha=\frac{1}{r}$, since also $r \leq 1$, implies that

$$
\begin{aligned}
\frac{1 \mathrm{~d} \zeta}{r} \frac{\mathrm{d} \sigma}{r} & =-\frac{4}{r}\left(1-\frac{1}{r}\right) f_{\Omega_{0}}\left|\nabla \chi^{\frac{1}{2}}\right|^{2}-\Phi(\sigma) \zeta(\sigma)+\Psi(\sigma) \frac{f_{\Omega_{0}} u^{p-1+r}}{\left(f_{\Omega_{0}} u^{r}\right)^{\gamma}} \\
& \geq-M_{\Phi} \zeta(\sigma)+m_{\Psi} \frac{\zeta^{2-\gamma}(\sigma)}{w(\sigma)} \geq \frac{\zeta(\sigma)}{w(\sigma)}\left(-M_{\Phi} w(\sigma)+m_{\Psi} \zeta^{1-\gamma}(\sigma)\right)
\end{aligned}
$$

which suffices by using (2.25) together with (2.1). Furthermore, since $\frac{p-1}{r}>1$, then (2.29) for $\alpha=\frac{1}{-p+1+r}$ leads to

$$
\alpha \frac{\mathrm{d} w}{\mathrm{~d} \sigma}=4 \alpha(\alpha-1) \int_{\Omega_{0}}\left|\nabla u^{\frac{1}{2 \alpha}}\right|^{2}-\Phi(\sigma) w+\Psi(\sigma) \zeta^{1-\gamma},
$$

which, owing to (2.1) and using the fact that $\alpha=\frac{1}{-p+1+r}<0$ ensures that

$$
\frac{1}{p-1-r} \frac{\mathrm{d} w}{\mathrm{~d} \sigma} \leq M_{\Phi} w(\sigma)-m_{\Psi} \zeta^{1-\gamma}(\sigma)
$$

Note that since $0<\gamma<1$, we have that the curve $\Gamma_{1}: w=\frac{m_{\Psi} \zeta^{1-\gamma}}{M_{\Phi}}, \zeta>0$, is concave in $w \zeta$-plane, with its endpoint at the origin $(0,0)$. Furthermore, relations $(2.30)$ and (2.32) imply that the region $\mathcal{R}=\left\{(\zeta, w) \mid w<\frac{m_{\Psi} \zeta^{1-\gamma}}{M_{\Phi}}\right\}$ is invariant, and $\zeta(\sigma)$ and $w(\sigma)$ are increasing and decreasing on $\mathcal{R}$, respectively. Under the assumption that $w(0)<\frac{m_{\Psi} \zeta^{1-\gamma}(0)}{M_{\Phi}}$, we have $\frac{\mathrm{d} w}{\mathrm{~d} \sigma}<0, \frac{\mathrm{d} \zeta}{\mathrm{d} \sigma}>0$, for $0 \leq \sigma<\Sigma$, and thus,

$$
\frac{m_{\Psi}}{w(\sigma)}-\frac{M_{\Phi}}{\zeta^{1-\gamma}(\sigma)} \geq \frac{m_{\Psi}}{w(0)}-\frac{M_{\Phi}}{\zeta^{1-\gamma}(0)} \equiv c_{0}>0, \text { for } 0 \leq \sigma<\Sigma .
$$


Therefore, by virtue of (2.30), we derive the differential inequality

$$
\begin{aligned}
\frac{1}{r} \frac{\mathrm{d} \zeta}{\mathrm{d} \sigma} \geq-M_{\Phi} \zeta(\sigma)+m_{\Psi} \frac{\zeta^{2-\gamma}(\sigma)}{w(\sigma)} & =\zeta^{2-\gamma}(\sigma)\left(\frac{1}{w(\sigma)}-\frac{M_{\Phi}}{m_{\Psi} \zeta^{1-\gamma}(\sigma)}\right) \\
& \geq c_{0} \zeta^{2-\gamma}(\sigma), \quad 0 \leq \sigma<\Sigma .
\end{aligned}
$$

Since $2-\gamma>1$, inequality (2.33) implies that $\zeta(\sigma)$ blows up in finite time $\sigma_{1} \leq \hat{\sigma}_{1} \equiv$ $\frac{\zeta^{\gamma-1}(0)}{(1-\gamma) c_{0} r}<\infty$, and since $\zeta(\sigma)=f_{\Omega_{0}} u^{r} \mathrm{~d} x \leq\|u(\cdot, \sigma)\|_{\infty}^{r}$ we conclude that $u(x, \sigma)$ blows up in finite time $\Sigma_{b} \leq \hat{\sigma}_{1}$.

We now consider the latter case when $\frac{p-1}{r} \geq 2$, then $q=\frac{p-1-r}{r} \geq 1$, and thus by virtue of Jensen's inequality, Evans (2010), we obtain $f_{\Omega_{0}} u^{r}\left(f_{\Omega_{0}}\left(u^{-r}\right)^{q}\right)^{\frac{1}{q}} \geq$ $f_{\Omega_{0}} u^{r} f_{\Omega_{0}} u^{-r} \geq 1$, which entails $\zeta^{\frac{1}{r}}(\sigma) \geq w^{-\frac{1}{p-1-r}}(\sigma)$, and thus by virtue of (2.1)

$$
w(\sigma) \geq \zeta^{-\frac{p-1-r}{r}(\sigma)}=\zeta^{1-\frac{p-1}{r}}(\sigma)>\frac{1}{\Phi(\sigma)} \zeta^{1-\frac{p-1}{r}}(\sigma) \geq \frac{m_{\Psi}}{M_{\Phi}} \zeta^{1-\frac{p-1}{r}}(\sigma),
$$

for any $\sigma \in[0, \Sigma)$. Since $\frac{p-1}{r} \geq 2$, the curve $\Gamma_{2}: w=\frac{m_{\Psi} \zeta^{1-\frac{p-1}{r}}}{M_{\Phi}}, \zeta>0$, is convex and approaches $+\infty$ and 0 as $\zeta \downarrow 0^{+}$and $\zeta \uparrow+\infty$, respectively. Moreover, the curves $\Gamma_{1}$ and $\Gamma_{2}$ intersect at the point $(\zeta, w)=(1,1)$, and therefore, $w(0)<1$ combined with (2.34) implies that $w(0)<\frac{m_{\Psi} \zeta^{1-\gamma}(0)}{M_{\Phi}}$. Thus, the latter case is reduced to the former case and once again finite-time blow-up for the solution $u(x, \sigma)$ is established.

Remark 2.7 Note that in the case of a stationary domain then $\zeta(\sigma)$ blows up, see Kavallaris and Suzuki $(2017,2018)$, in finite time $\sigma_{2} \leq \hat{\sigma}_{2} \equiv \frac{\zeta^{\gamma-1}(0)}{(1-\gamma) c_{1} r}$, where $c_{1} \equiv$ $\frac{1}{w(0)}-\frac{1}{\zeta^{1-\gamma}(0)}$, and thus $u(x, \sigma)$ blows in finite time $\Sigma_{1} \leq \hat{\sigma}_{2}$ under the condition $w(0)<\zeta(0)^{1-\gamma}$.

Remark 2.8 For a logistically growing or shrinking domain problem, (1.32)-(1.34) exhibit finite-time blow-up under the assumptions of Theorem 2.2 whenever $w(0)<$ $M_{L}^{-(\gamma+1)} \zeta(0)^{1-\gamma}$, where $M_{L}:=\sup _{(0, \infty)} L(t)=\sup _{(0, \infty)}\left(1+\frac{N \beta\left(1-\frac{1}{m}\right)}{1+\frac{1}{m}\left(\mathrm{e}^{\beta t}-1\right)}\right)$. In particular, for a logistically growing domain, when $m>1$, then $M_{L}=L(0)=$ $1+N \beta\left(1-\frac{1}{m}\right)$, whilst for logistically decaying domain, when $0<m<1$ we have $M_{L}=\lim _{t \rightarrow+\infty} L(t)=1$ and hence in that case blow-up conditions (1) and (2) of Theorem 2.2 coincide with the ones of Kavallaris and Suzuki (2017, Theorem 3.5), see also Remark 2.7.

Now, we present a global-in-time existence result stated as follows.

Theorem 2.3 Assume that $\frac{p-1}{r}<\min \left\{1, \frac{2}{N}, \frac{1}{2}\left(1-\frac{1}{r}\right)\right\}$ and $0<\gamma<1$. Consider functions $\Phi(\sigma), \Psi(\sigma)>0$ with

$$
\inf _{(0, \Sigma)} \Phi(\sigma):=m_{\Phi}>0 \text { and } \sup _{(0, \Sigma)} \Psi(\sigma):=M_{\Psi}<+\infty
$$


then problem (1.35)-(1.37) has a global-in-time solution.

Proof We assume $\frac{p-1}{r}<\min \left\{1, \frac{2}{N}, \frac{1}{2}\left(1-\frac{1}{r}\right)\right\}$ and $0<\gamma<1$. We also assume $N \geq 2$ since the complementary case $N=1$ is simpler.

Note that for $p>1$, we have $\frac{p-1}{r}<\frac{2}{N}$ and $r>p$. Therefore, we have $0<$ $\frac{1}{r-p+1}<\min \left\{1,\left(\frac{1}{p-1}\right)\left(\frac{2}{N-2}\right), \frac{1}{1-p+r \gamma}\right\}$, since $0<\gamma<1$. Choosing $\frac{1}{r-p+1}<$ $\alpha<\min \left\{1, \frac{1}{p-1} \cdot \frac{2}{N-2}, \frac{1}{1-p+r \gamma}\right\}$, it follows that the $\max \left\{\frac{N-2}{N}, \frac{1}{\alpha r}\right\}<\frac{1}{-\alpha+1+\alpha p}$, and then we can find $\beta>0$ such that

$$
\max \left\{\frac{N-2}{N}, \frac{1}{\alpha r}\right\}<\frac{1}{\beta}<\frac{1}{-\alpha+1+\alpha p}<2,
$$

which satisfies

$$
\frac{\beta}{\alpha r}<1<\frac{\beta}{-\alpha+1+\alpha p}
$$

Recalling that $\chi=u^{\frac{1}{\alpha}}$ satisfies (2.26)-(2.28) with $\int_{\Omega_{0}} \frac{u^{p-1+\frac{1}{\alpha}}}{\left(f_{\Omega_{0}} u^{r}\right)^{\gamma}}=\frac{f_{\Omega_{0}} \chi^{-\alpha+1+\alpha p}}{\left(f_{\Omega_{0}} \chi^{\alpha r}\right)^{\gamma}}$, then by virtue of (2.37)

$$
f_{\Omega_{0}} \chi^{-\alpha+1+\alpha p} \leq\left(f_{\Omega_{0}} \chi^{\beta}\right)^{\frac{-\alpha+1+\alpha p}{\beta}} \text { and }\left(f_{\Omega_{0}} \chi^{\alpha r}\right)^{\gamma} \geq\left(f_{\Omega_{0}} \chi^{\beta}\right)^{\frac{\alpha r}{\beta} \cdot \gamma},
$$

thus we obtain the following estimate

$$
\frac{f_{\Omega_{0}} \chi^{-\alpha+1+\alpha p}}{\left(f_{\Omega_{0}} \chi^{\alpha r}\right)^{\gamma}} \leq\left(f_{\Omega_{0}} \chi^{\beta}\right)^{\frac{-\alpha+1+\alpha p-\alpha r \gamma}{\beta}}=\left\|\chi^{\frac{1}{2}}\right\|_{2 \beta}^{2(1-\lambda)},
$$

with $0<\lambda=\alpha\{1-p+r \gamma\}<1$, recalling that $\frac{p-1}{r}<\gamma$ and $\alpha<\frac{1}{1-p+r \gamma}$. Averaging (2.26) over $\Omega_{0}$ leads to the following,

$$
\alpha \frac{d}{d \sigma} f_{\Omega_{0}} \chi+4 \alpha(1-\alpha) f_{\Omega_{0}}\left|\nabla \chi^{\frac{1}{2}}\right|^{2}+\Phi(\sigma) f_{\Omega_{0}} \chi=\Psi(\sigma) \frac{f_{\Omega_{0}} \chi^{-\alpha+1+\alpha p}}{\left(f_{\Omega_{0}} \chi^{\alpha r}\right)^{\gamma}}
$$

and hence

$$
\frac{d}{d \sigma} \int_{\Omega_{0}} \chi+4(1-\alpha) f_{\Omega_{0}}\left|\nabla \chi^{\frac{1}{2}}\right|^{2}+\frac{m_{\Phi}}{\alpha} f_{\Omega_{0}} \chi \leq \frac{M_{\Psi}}{\alpha}\left\|\chi^{\frac{1}{2}}\right\|_{2 \beta}^{2(1-\lambda)},
$$

by virtue of (2.35), (2.36) and (2.38). Now since $1<2 \beta<\frac{2 N}{N-2}$ holds due to (2.36) and applying first the Sobolev's and then Young's inequalities we obtain

$$
\frac{d}{d \sigma} f_{\Omega_{0}} \chi+c\left\|\chi^{\frac{1}{2}}\right\|_{H^{1}}^{2}+\frac{M_{\Psi}}{\alpha} f_{\Omega_{0}} \chi \leq C
$$


which implies $f_{\Omega_{0}} \chi \leq C$. Since $\frac{1}{\alpha}$ can be chosen to be close to $r-p+1$, the above estimate gives

$$
\|u(\cdot, \sigma)\|_{q} \leq C_{q}, \quad \text { for any } \quad 1 \leq q<r-p+1,
$$

recalling that $\chi=u^{\frac{1}{\alpha}}$. Note that $\frac{p-1}{r}<\frac{1}{2}\left(1-\frac{1}{r}\right)$ implies $\frac{r-p+1}{p}>1$ and thus we obtain global-in-time existence by using the same bootstrap argument as in Kavallaris and Suzuki (2017, Theorem 3.4).

Remark 2.9 Note that condition (2.35) is satisfied in the case of an exponentially shrinking domain as indicated in Remark 2.4, see in particular (2.19) and (2.20).

\section{Turing Instability and Pattern Formation}

In this section, we state and prove a Turing-instability, that is a diffusion-driven instability, result for problem (1.35)-(1.37). Due to technical restrictions, we focus on the radial case $\Omega_{0}=B_{1}(0):=\left\{x \in \mathbb{R}^{N}|| x \mid<1\right\}$ and for dimensions $N \geq 3$; however, in Sect. 4, we treat numerically the two-dimensional case $N=2$ as well, see Fig. 6 Next, we consider a radially decreasing and spiky initial datum of the form, (Hu and Yin 1995),

$$
u_{0}(R)=\lambda \psi_{\delta}(R)
$$

with $0<\lambda \ll 1$ and

$$
\psi_{\delta}(R)= \begin{cases}R^{-a}, & \delta \leq R \leq 1 \\ \delta^{-a}\left(1+\frac{a}{2}\right)-\frac{a}{2} \delta^{-(a+2)} R^{2}, & 0 \leq R<\delta\end{cases}
$$

where $a=\frac{2}{p-1}$ and $0<\delta<1$. Notably $u_{0}(R) \in L^{\infty}(0,1)$, which is compatible with assumption (1.10).

Then, the solution $u$ (1.35)-(1.37) is radially symmetric and decreasing, i.e. $u(x, \sigma)=u(R, \sigma)$ for $R=|x|$ and $u_{R}(R, \sigma) \leq 0$ and thus, it satisfies the following

$$
\begin{aligned}
u_{\sigma} & =\Delta_{R} u-\Phi(\sigma) u+\frac{\Psi(\sigma) u^{p}}{\left(f_{\Omega_{0}} u^{r}\right)^{\gamma}}, \quad R \in(0,1), \sigma \in(0, \Sigma), \\
u_{R}(0, \sigma) & =u(1, \sigma)=0, \quad \sigma \in(0, \Sigma), \\
u(R, 0) & =u_{0}(R), \quad 0<R<1,
\end{aligned}
$$

where $\Delta_{R} u:=u_{R R}+\frac{N-1}{R} u_{R}$.

Remarkably, under the Turing condition (1.5), the spatial homogeneous solutions of (3.3)-(3.5), i.e. the solution of the problem

$$
\begin{aligned}
\frac{\mathrm{d} u}{\mathrm{~d} \sigma} & =-\Phi(\sigma) u+\Psi(\sigma) u^{p-r \gamma}, \\
\left.u\right|_{\sigma=0} & =\bar{u}_{0}>0,
\end{aligned}
$$


never exhibit blow-up, as long as $\Phi(\sigma), \Psi(\sigma)$ are both bounded, since the nonlinearity $f(u)=u^{p-r \gamma}$ is sub-linear [see also Kavallaris and Suzuki $\left.(2017,2018)\right]$. Otherwise, considering spatial inhomogeneous solutions of (3.3)-(3.5), the following diffusiondriven blow-up (Turing instability) result holds true.

Theorem 3.1 Consider $N \geq 3,1 \leq r \leq p, p>\frac{N}{N-2} E, \frac{2}{N}<\frac{p-1}{r}<\gamma$ and $\gamma>1$. Assume that both $\Phi(\sigma)$ and $\Psi(\sigma)$ are positive and bounded. Then, there exists $\lambda_{0}>0$ such that for any $0<\lambda \leq \lambda_{0}$ there exists $0<\delta_{0}=\delta_{0}(\lambda)<1$, then any solution of problem (3.3)-(3.5) with spiky initial data of the form (3.1) and $0<\delta \leq \delta_{0}$ blows up in finite time.

Note that the maximum principle is not applicable for the non-local problem (3.3)(3.5) and hence comparison techniques fail, see for example Quittner and Souplet (2007, Proposition 52.24). Therefore, to overcome this obstacle, and finally prove Theorem 3.1, we derive a lower estimate of the non-local term

$$
\widetilde{K}(\sigma):=\frac{\Psi(\sigma)}{\left(f_{\Omega_{0}} u^{r}\right)^{\gamma}}
$$

and then deal with a local problem for which comparison techniques become applicable. To that end, following an approach used in Hu and Yin (1995) and Kavallaris and Suzuki $(2017,2018)$, we need to prove first some auxiliary results.

First, it is easily seen that for $\psi_{\delta}$ given by (3.2) the following lemma holds (Kavallaris and Suzuki 2017, 2018).

Lemma 3.1 For the function $\psi_{\delta}$ defined by (3.2), we have:

(i) For any $0<\delta<1$, there holds in a weak sense

$$
\Delta_{R} \psi_{\delta} \geq-N a \psi_{\delta}^{p}
$$

(ii) If $m>0$ and $N>m a$, we have

$$
f_{\Omega_{0}} \psi_{\delta}^{m}=\frac{N}{N-m a}+O\left(\delta^{N-m a}\right), \quad \delta \downarrow 0 .
$$

Now, if we consider

$$
\mu>1+r \gamma
$$

and set $\alpha_{1}=\sup _{0<\delta<1} \frac{1}{\bar{\psi}_{\delta}^{\mu}} f_{\Omega_{0}} \psi_{\delta}^{p}$, and $\alpha_{2}=\inf _{0<\delta<1} \frac{1}{\bar{\psi}_{\delta}^{\mu}} f_{\Omega_{0}} \psi_{\delta}^{p}$,

then since $p>\frac{N}{N-2}$, relation (3.7) is applicable for $m=p$ and $m=1$, and thus owing to (3.8) we obtain

$$
0<\alpha_{1}, \alpha_{2}<\infty
$$


Furthermore, it follows that

$$
d \equiv \inf _{0<\delta<1}\left(\frac{1}{2 \alpha_{1}}\right)^{\frac{r \gamma}{p}}\left(\frac{1}{2 \bar{\varphi}_{\delta}}\right)^{\frac{r \gamma}{p} \mu}>0
$$

and the initial data $u_{0}$ defined by (3.1) and (3.2) also satisfy the following lemma, for the proof see Kavallaris and Suzuki (2017, 2018).

Lemma 3.2 If $p>\frac{N}{N-2}$ and $\frac{p-1}{r}<\gamma$, there exists $\lambda_{0}=\lambda_{0}(d)>0$ such that for any $0<\lambda \leq \lambda_{0}$ there holds

$$
\Delta_{R} u_{0}+d \lambda^{-r \gamma} u_{0}^{p} \geq 2 u_{0}^{p} .
$$

Hereafter, we fix $0<\lambda \leq \lambda_{0}=\lambda_{0}(d)$ such that (3.11) is satisfied. Given $0<\delta<1$, let $\Sigma_{\delta}>0$ be the maximal existence time of the solution to (3.3)-(3.5) with initial data of the form (3.1)-(3.2). Next, we introduce the new variable $z:=\mathrm{e}^{\int^{\sigma} \Phi(s) d s} u$, such that the linear dissipative term $-\Phi(\sigma) u$ in (3.3) is eliminated and $z$ satisfies

$$
\begin{aligned}
z_{\sigma}= & \Delta_{R} z \\
& +K(\sigma) z^{p}, \quad R \in(0,1), \sigma \in\left(0, \Sigma_{\delta}\right), \\
z_{R}(0, \sigma)= & z_{R}(1, \sigma)=0, \quad \sigma \in\left(0, \Sigma_{\delta}\right), \\
z(R, 0)= & u_{0}(R), \quad 0<R<1,
\end{aligned}
$$

where

$$
K(\sigma):=\frac{\Psi(\sigma) \mathrm{e}^{(1+r \gamma-p) \int^{\sigma} \Phi(s) d s}}{\left(f_{\Omega_{0}} z^{r}\right)^{\gamma}} .
$$

It is clear that as long as $\Phi(\sigma)$ is bounded then $u$ blows-up in finite time if and only if $z$ does so. Assuming now that both $\Phi(\sigma)$ and $\Psi(\sigma)$ are positive and bounded, which is the case for the evolution provided by $\psi(\sigma)$ satisfying (2.3) or for an exponentially shrinking domain as indicated in Remarks 2.1 and 2.4, then by virtue of (2.14) we have

$$
0<K(\sigma)=\frac{\Psi(\sigma) \mathrm{e}^{(1-p) \int^{\sigma} \Phi(s) d s}}{\left(f_{\Omega_{0}} u^{r}\right)^{\gamma}} \leq C<\infty .
$$

Averaging of (3.12) entails

$$
\frac{d \bar{z}}{d \sigma}=K(\sigma) f_{\Omega_{0}} z^{p}
$$

and thus (3.16) yields

$$
\bar{z}(\sigma) \geq \bar{z}(0)=\bar{u}_{0}:=\oint_{\Omega_{0}} u_{0} .
$$

Henceforth, the positivity and the boundedness of $\Phi(\sigma)$, and $\Psi(\sigma)$ as well as the Turing condition (1.5) are imposed. 
Next, we provide a useful estimate of $z$ that will be frequently used throughout the sequel.

Lemma 3.3 The solution $z$ of problem (3.12)-(3.14) satisfies

$$
\begin{aligned}
& R^{N} z(R, \sigma) \leq \bar{z}(\sigma) \text { in }(0,1) \times\left(0, \Sigma_{\delta}\right), \\
& \text { and } \\
& z_{R}\left(\frac{3}{4}, \sigma\right) \leq-c, \quad 0 \leq \sigma<\Sigma_{\delta}
\end{aligned}
$$

for any $0<\delta<1$ and some positive constant $c$.

Proof Let us define $w=R^{N-1} z_{R}$, then it follows that $w$ satisfies $\mathcal{H}[w]=$ 0 , for $(R, \sigma) \in(0,1) \times\left(0, \Sigma_{\delta}\right)$, with $w(0, \sigma)=w(1, \sigma)=0$, for $\sigma \in\left(0, \Sigma_{\delta}\right)$, and $w(R, 0)<0$, for $0<R<1$, where $\mathcal{H}[w] \equiv w_{\sigma}-w_{R R}+\frac{N-1}{\rho} w_{R}-p K(\sigma) z^{p-1} w$. Owing to the maximum principle, and recalling that $K(\sigma)$ is bounded by (3.16), we get that $w \leq 0$, which implies $z_{R} \leq 0$ in $(0,1) \times\left(0, \Sigma_{\delta}\right)$. Accordingly, inequality (3.19) follows since

$$
\begin{aligned}
R^{N} z(R, \sigma) & =z(R, \sigma) \int_{0}^{R} N s^{N-1} \mathrm{~d} s \leq \int_{0}^{R} N z(s, \sigma) s^{N-1} \mathrm{~d} s \\
& \leq \int_{0}^{1} N z(s, \sigma) s^{N-1} \mathrm{~d} s=\int_{\Omega_{0}} z=\bar{z}(\sigma) .
\end{aligned}
$$

Now, given that $w \leq 0$ together with (3.16), we have

$$
w_{\sigma}-w_{R R}+\frac{N-1}{\rho} w_{R}=p K(\sigma) z^{p-1} w \leq 0 \text { in }(0,1) \times\left(0, \Sigma_{\delta}\right),
$$

with $w\left(\frac{1}{2}, \sigma\right) \leq 0, \quad w(1, \sigma) \leq 0$, for $\sigma \in\left(0, \Sigma_{\delta}\right)$, and $w(R, 0)=\rho^{N-1} u_{0}^{\prime}(R) \leq$ $-c$, for $\frac{1}{2}<\rho<1$, which implies $w \leq-c$ in $\left(\frac{1}{2}, 1\right) \times\left(0, \Sigma_{\delta}\right)$, and thus (3.20) holds.

The next result is vital for proving the key estimate provided by Proposition 3.1 below.

Lemma 3.4 Take $\varepsilon>0$ and $1<q<p$ then $\vartheta$ defined as

$$
\vartheta:=R^{N-1} z_{R}+\varepsilon \cdot \frac{R^{N} z^{q}}{\bar{z}^{\gamma+1}}
$$

satisfies

$$
\begin{aligned}
\mathcal{H}[\vartheta] \leq- & \frac{2 q \varepsilon}{\bar{z}^{\gamma+1}} z^{q-1} \vartheta+\frac{\varepsilon R^{N} z^{q}}{\bar{z}^{2(\gamma+1)}}\left\{2 q \varepsilon z^{q-1}\right. \\
& \left.\quad-m_{\Psi}(\gamma+1) \bar{z}^{\gamma-r \gamma} \int_{\Omega_{0}} z^{p}-m_{\Psi}(p-q) z^{p-1} \bar{z}^{\gamma+1-r \gamma}\right\},
\end{aligned}
$$

for $(R, \sigma) \in(0,1) \times\left(0, \Sigma_{\delta}\right)$, where $m_{\Psi}=\inf _{\sigma \in\left(0, \Sigma_{\delta}\right)} \Psi(\sigma)>0$. 
Proof It is readily checked that $\mathcal{H}\left[R^{N-1} z_{R}\right]=0$, while by straightforward calculations we derive

$$
\begin{aligned}
& \mathcal{H}\left[\varepsilon R^{N} \frac{z^{q}}{\bar{z}^{\gamma+1}}\right]=\frac{2 q(N-1) \varepsilon R^{N-1} z^{q-1}}{\bar{z}^{\gamma+1}} z_{R} \\
& +\frac{q \varepsilon R^{N} z^{p-1+q}}{\bar{z}^{\gamma+1}} K(\sigma) \\
& -\frac{(\gamma+1) \varepsilon R^{N} z^{q}}{\bar{z}^{\gamma+2}} K(\sigma) f_{\Omega_{0}} z^{p} \mathrm{~d} x \\
& -\frac{2 q N \varepsilon R^{N-1} z^{q-1}}{\bar{z}^{\gamma+1}} z_{R} \\
& -\frac{q(q-1) \varepsilon R^{N} z^{q-2}}{\bar{z}^{\gamma+1}} z_{R}^{2}-\frac{p \varepsilon R^{N} z^{p-1+q}}{\bar{z}^{\gamma+1}} K(\sigma) \\
& \leq-\frac{2 q \varepsilon z^{q-1}}{\bar{z}^{\gamma+1}} \vartheta \\
& +\frac{\varepsilon R^{N} z^{q}}{\bar{z}^{2(\gamma+1)}}\left[2 q \varepsilon z^{q-1}-\frac{\Psi(\sigma)(\gamma+1) \bar{z}^{\gamma} \mathrm{e}^{(1+r \gamma-p) \int^{\sigma} \Phi(s) d s}}{\left(f_{\Omega_{0}} z^{r} \mathrm{~d} x\right)^{\gamma}} \int_{\Omega_{0}} z^{p} \mathrm{~d} x\right. \\
& \left.-\frac{\Psi(\sigma)(p-q) z^{p-1} \bar{z}^{\gamma+1}}{\left(f_{\Omega_{0}} z^{r} \mathrm{~d} x\right)^{\gamma}} \mathrm{e}^{(1+r \gamma-p) \int^{\sigma} \Phi(s) \mathrm{d} s}\right] \text {. }
\end{aligned}
$$

Then, by virtue of the Hölder's inequality, and since $1 \leq r \leq p$, (3.23) entails the desired estimate (3.22).

Next, note that when $p>\frac{N}{N-2}$, there is $1<q<p$ such that $N>\frac{2 p}{q-1}$ and thus the following quantities

$$
A_{1} \equiv \sup _{0<\delta<1} \frac{1}{\bar{u}_{0}^{\mu}} \int_{\Omega_{0}} u_{0}^{p}=\lambda^{\mu-p} \alpha_{1} \quad \text { and } \quad A_{2} \equiv \inf _{0<\delta<1} \frac{1}{\bar{u}_{0}^{\mu}} \int_{\Omega_{0}} u_{0}^{p}=\lambda^{\mu-p} \alpha_{2} \text {, }
$$

are finite due to (3.9).

An essential ingredient for the proof of Theorem 3.1 is the following key estimate of the $L^{p}-$ norm of $z$ in terms of $A_{1}$ and $A_{2}$.

Proposition 3.1 There exist $0<\delta_{0}<1$ and $0<\sigma_{0} \leq 1$ independent of any $0<\delta \leq$ $\delta_{0}$, such that the following estimate is satisfied

$$
\frac{1}{2} A_{2} \bar{z}^{\mu} \leq \int_{\Omega_{0}} z^{p} \mathrm{~d} x \leq 2 A_{1} \bar{z}^{\mu}
$$

for any $0<\sigma<\min \left\{\sigma_{0}, \Sigma_{\delta}\right\}$.

The proof of Proposition 3.1 requires some further auxiliary results provided below. Let us define $0<\sigma_{0}(\delta)<\Sigma_{\delta}$ to be the maximal time for which inequality (3.25) is 
valid in $0<\sigma<\sigma_{0}(\delta)$, then we have

$$
\frac{1}{2} A_{2} \bar{z}^{\mu} \leq \int_{\Omega_{0}} z^{p} \leq 2 A_{1} \bar{z}^{\mu}
$$

We only regard the case $\sigma_{0}(\delta) \leq 1$, since otherwise there is nothing to prove. Then, the following lemma holds true.

Lemma 3.5 There exists $0<\sigma_{1}<1$ such that

$$
\bar{z}(\sigma) \leq 2 \bar{u}_{0}, \quad 0<\sigma<\min \left\{\sigma_{1}, \sigma_{0}(\delta)\right\}
$$

for any $0<\delta<1$.

Proof Since $r \geq 1$ and $\sigma_{0}(\delta) \leq 1$, then by virtue of (3.15) and (3.17)

$$
\frac{\mathrm{d} \bar{z}}{\mathrm{~d} \sigma} \leq 2 A_{1} M_{\Psi} \mathrm{e}^{(1+r \gamma-p) M_{\Phi}} \bar{z}^{\mu-r \gamma}, \quad \text { for } 0<\sigma<\sigma_{0}(\delta),
$$

recalling that $M_{\Phi}=\sup _{\sigma \in\left(0, \Sigma_{\delta}\right)} \Phi(\sigma)<+\infty$ and $M_{\Psi}=\sup _{\sigma \in\left(0, \Sigma_{\delta}\right)} \Psi(\sigma)<+\infty$.

Setting $C_{1}=2 A_{1} M_{\Psi} \mathrm{e}^{(1+r \gamma-p) M_{\Phi}}$ and taking into account (3.8), we then derive

$$
\bar{z}(\sigma) \leq\left[\bar{u}_{0}^{1+r \gamma-\mu}-C_{1}(\mu-r \gamma-1) \sigma\right]^{-\frac{1}{\mu-r \gamma-1}} .
$$

Accordingly, (3.27) holds for any $0<\sigma<\min \left\{\sigma_{1}, \sigma_{0}(\delta)\right\}$ where $\sigma_{1}$ is independent of any $0<\delta<1$ and is estimated as $\sigma_{1} \leq \min \left\{\frac{1-2^{1+r \gamma-\mu}}{C_{1}(\mu-r \gamma-1)} \bar{u}_{0}^{1+r \gamma-\mu}, 1\right\}$.

Another fruitful estimate is provided by the next lemma.

Lemma 3.6 There exist $0<\delta_{0}<1$ and $0<R_{0}<\frac{3}{4}$ such that for any $0<\delta \leq \delta_{0}$ the following estimate is valid

$$
\frac{1}{|\Omega|} \int_{B_{R_{0}}(0)} z^{p} \leq \frac{A_{2}}{8} \bar{z}^{\mu}, \text { for } 0<\sigma<\min \left\{\sigma_{1}, \sigma_{0}(\delta)\right\},
$$

where $B_{R_{0}}(0)=\left\{x \in \mathbb{R}^{N}|| x \mid<R_{0}\right\}$.

Proof By virtue of (3.18) and (3.27), it follows that

$$
\bar{u}_{0} \leq \bar{z}(\sigma) \leq 2 \bar{u}_{0}, \quad \text { for } \quad 0<\sigma<\min \left\{\sigma_{1}, \sigma_{0}(\delta)\right\}
$$

Furthermore, we note that the growth of $f_{\Omega_{0}} z^{p}$ is controlled by the estimate (3.25) for $0<\min \left\{\sigma_{1}, \sigma_{0}(\delta)\right\}$ and since $p>q$ then Young's inequality ensures that the second term of the right-hand side in (3.22) is negative for $0<\sigma<\min \left\{\sigma_{1}, \sigma_{0}(\delta)\right\}$, uniformly in $0<\delta<1$, provided that $0<\varepsilon \leq \varepsilon_{0}$ for some $0<\varepsilon_{0} \ll 1$. Therefore,

$$
\mathcal{H}[\vartheta] \leq-\frac{2 q \varepsilon z^{q-1}}{\bar{z}^{\gamma+1}} \vartheta \quad \text { in } \quad(0,1) \times\left(0, \min \left\{\sigma_{1}, \sigma_{0}(\delta)\right\}\right)
$$


Moreover, (3.19) and (3.29) imply

$$
\begin{aligned}
\vartheta(R, \sigma) & =R^{N-1} z_{R}+\varepsilon \frac{R^{N} z^{q}}{\bar{z}^{\gamma+1}} \leq R^{N-1} z_{R}+\varepsilon R^{N(1-q)} \bar{z}^{q-\gamma-1} \\
& \leq R^{N-1} z_{R}+C \varepsilon R^{N(1-q)} \text { in }(0,1) \times\left(0, \min \left\{\sigma_{1}, \sigma_{0}(\delta)\right\}\right),
\end{aligned}
$$

which, for $0<\varepsilon \leq \varepsilon_{0}$, entails

$$
\vartheta\left(\frac{3}{4}, \sigma\right)<0, \quad 0<\sigma<\min \left\{\sigma_{1}, \sigma_{0}(\delta)\right\}
$$

owing to (3.20) and provided that $0<\varepsilon_{0} \ll 1$. Additionally, (3.21) for $t=0$ gives

$$
\vartheta(R, 0)=R^{N-1}\left(\lambda \psi_{\delta}^{\prime}(R)+\varepsilon \lambda^{q-\gamma-1} R \frac{\psi_{\delta}^{q}}{\bar{\psi}_{\delta}^{\gamma+1}}\right)
$$

For $0 \leq R<\delta$ and $\varepsilon$ small enough and independent of $0<\delta<\delta_{0}$, then the right-hand side of (3.32) can be estimated as

$$
R^{N} \lambda\left(-a \delta^{-a-2}+\varepsilon \lambda^{q-\gamma-2} \frac{\psi_{\delta}^{q}}{\bar{\psi}_{\delta}^{\gamma+1}}\right) \lesssim R^{N} \lambda\left(-a \delta^{-a-2}+\varepsilon \lambda^{q-\gamma-2} \delta^{-a q}\right) \lesssim 0
$$

since by virtue of (3.2) and (3.7) and for $m=1$, there holds $\frac{\psi_{\delta}^{q}}{\bar{\psi}_{\delta}^{\gamma+1}} \lesssim \delta^{-a q}, \delta \downarrow 0$, uniformly in $0 \leq R<\delta$, taking also into account that $a+2=a p>a k$.

On the other hand, for $\delta \leq R \leq 1$ and by using (3.7) for $m=1$ we take

$$
\vartheta(R, 0)=R^{N} \lambda\left(-a R^{-a-1}+\varepsilon \lambda^{q-\gamma-1} \frac{R^{1-a q}}{\bar{\psi}_{R}^{\gamma+1}}\right)
$$

which, since $a+2=a p>a q$ implies $-a-1<-a q+1$, finally yields $\vartheta(R, 0)<$ $0, \quad \delta \leq R \leq \frac{3}{4}$, for any $0<\delta \leq \delta_{0}$ and $0<\varepsilon \leq \varepsilon_{0}$, provided $\varepsilon_{0}$ is chosen sufficiently small. Accordingly, it follows that

$$
\vartheta(R, 0)<0, \quad \text { and } \quad 0 \leq R \leq \frac{3}{4}
$$

for any $0<\delta \leq \delta_{0}$ and $0<\varepsilon \leq \varepsilon_{0}$, provided $0<\varepsilon_{0} \ll 1$.

In conjunction of (3.30), (3.31) and (3.34), we deduce $\vartheta(R, \sigma)=R^{N-1} z_{R}+$ $\varepsilon \frac{R^{N} z^{q}}{\bar{z}^{\gamma+1}} \leq 0$ in $\left(0, \frac{3}{4}\right) \times\left(0, \min \left\{\sigma_{1}, \sigma_{0}(\delta)\right\}\right)$, and finally

$$
z(R, \sigma) \leq\left(\frac{\varepsilon}{2}(q-1)\right)^{-\frac{1}{q-1}} R^{-\frac{2}{q-1} z^{\frac{\gamma-1}{q-1}}}(\sigma) \text { in }\left(0, \frac{3}{4}\right) \times\left(0, \min \left\{\sigma_{1}, \sigma_{0}(\delta)\right\}\right) .
$$


Note that owing to $N>\frac{2 p}{q-1}$ there holds $-\left(\frac{2}{q-1}\right) p+N-1>-1$ and thus (3.28) is valid for some $0<R_{0}<\frac{3}{4}$.

Remark 3.1 Estimate (3.35) entails that $z(R, \sigma)$ can only blow-up in the origin $R=0$; that is, only a single-point blow-up is feasible.

Next, we prove the key estimate (3.25) using essentially Lemmas 3.5 and 3.6.

Proof of Proposition 3.1 By virtue of (3.8) and since $\frac{p-1}{r}<\delta$, there holds that $\ell=$ $\frac{\mu}{p}>1$. We can easily check that $\theta=\frac{z}{\bar{z}^{\ell}}$ satisfies (Kavallaris and Suzuki 2017, 2018)

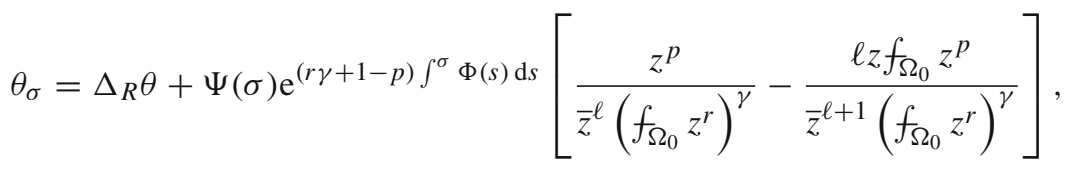

in $\Omega_{0} \times\left(0, \min \left\{\sigma_{0}, \Sigma_{\delta}\right\}\right)$, with $\frac{\partial \theta}{\partial v}=0$, on $\partial \Omega_{0} \times\left(0, \min \left\{\sigma_{0}, \Sigma_{\delta}\right\}\right)$, and $\theta(x, 0)=$ $\frac{z(x, 0)}{\bar{z}_{0}^{\ell}}$, on $\Omega_{0}$. In conjunction with (2.14), (3.18), (3.19), (3.26), and (3.27), we deduce that

$$
\left\|\theta, \frac{z^{p}}{\bar{z}^{\ell}\left(f_{\Omega_{0}} z^{r}\right)^{\gamma}}, \frac{\ell z f_{\Omega_{0}} z^{p}}{\bar{z}^{\ell+1}\left(f_{\Omega_{0}} z^{r}\right)^{\gamma}}\right\|_{L^{\infty}\left(\left(\Omega_{0} \backslash B_{R_{0}}(0)\right) \times \min \left\{\sigma_{1}, \sigma_{0}(\delta)\right\}\right)}<+\infty,
$$

uniformly in $0<\delta \leq \delta_{0}$, and using the fact that $\Psi(\sigma)$ and $\Psi(\sigma)$ are both bounded and positive. Estimate (3.36) according to the standard parabolic regularity condition, see DeGiorgi-Nash-Moser estimates in Lieberman (1996, pp. 144-145), entails the existence of $0<\sigma_{2} \leq \sigma_{1}$ independent of $0<\delta \leq \delta_{0}$ : $\sup _{0<\sigma<\min \left\{\sigma_{2}, \sigma_{0}(\delta)\right\}}\left\|\theta^{p}(\cdot, \sigma)-\theta^{p}(\cdot, 0)\right\|_{L^{1}\left(\Omega_{0} \backslash B_{R_{0}}(0)\right.} \leq \frac{A_{2}}{8}\left|\Omega_{0}\right|$, which yields

$$
\left|\frac{1}{\left|\Omega_{0}\right|} \int_{\Omega_{0} \backslash B_{R_{0}}(0)} \frac{z^{p}}{\bar{z}^{\mu}}-\frac{1}{\left|\Omega_{0}\right|} \int_{\Omega_{0} \backslash B_{R_{0}}(0)} \frac{z_{0}^{p}}{\bar{z}_{0}^{\mu}}\right| \leq \frac{A_{2}}{8},
$$

with $0<\sigma<\min \left\{\sigma_{2}, \sigma_{0}(\delta)\right.$ for any $0<\delta \leq \delta_{0}$. Combining (3.28) and (3.37) we deduce $\left|f_{\Omega_{0}} \frac{z^{p}}{\bar{z}^{\mu}}-f_{\Omega_{0}} \frac{z_{0}^{p}}{\bar{z}_{0}^{\mu}}\right| \leq \frac{3 A_{2}}{8}$, for $0<\sigma<\min \left\{\sigma_{2}, \sigma_{0}(\delta)\right\}$ and $0<\delta \leq \delta_{0}$, and thus, we finally obtain

$$
\frac{5 A_{2}}{8} \leq f_{\Omega_{0}} \frac{z^{p}}{\bar{z}^{\mu}} \leq \frac{11 A_{1}}{8}, \text { for } 0<\sigma<\min \left\{\sigma_{2}, \sigma_{0}(\delta)\right\}, 0<\delta \leq \delta_{0}
$$

taking also into consideration $A_{2} \leq f_{\Omega_{0}} \frac{z_{0}^{p}}{\bar{z}_{0}^{\mu}} \leq A_{1}$. Consequently, if we consider $\sigma_{0}(\delta) \leq \sigma_{2}$, then it follows that $\frac{1}{2} A_{2} \bar{z}^{\mu}<\frac{5}{8} A_{2} \bar{z}^{\mu} \leq f_{\Omega_{0}} z^{p} \leq \frac{11}{8} A_{1} \bar{z}^{\mu}<2 A_{1} \bar{z}^{\mu}$, 
for $0<\sigma<\sigma_{0}(\delta)$, and thus a continuity argument implies that $\frac{1}{2} A_{2} \bar{z}^{\mu} \leq f_{\Omega_{0}} z^{p} \leq$ $2 A_{1} \bar{z}^{\mu}$, with $0<\sigma<\sigma_{0}(\delta)+\eta$, for some $\eta>0$, which contradicts the definition of $\sigma_{0}(\delta)$. Accordingly, we derive that $\sigma_{2}<\sigma_{0}(\delta)$ for any $0<\delta \leq \delta_{0}$, and the proof of Proposition 3.1 is complete for $\sigma_{0}=\sigma_{2}$.

Now, we are ready to proceed with the proof of Theorem 3.1.

Proof of Theorem 3.1 First, note that $\sigma_{0} \leq \sigma_{1}$ in (3.27), then from (3.10) and (3.24), we have

$$
K(\sigma) \geq \frac{m_{\Psi}}{\left(f_{\Omega_{0}} z^{p}\right)^{\frac{r \gamma}{p}}} \geq m_{\Psi}\left(\frac{1}{2 \alpha_{1}}\right)^{\frac{r \gamma}{p}} \cdot\left(\frac{1}{2 \bar{\psi}_{\delta}}\right)^{\frac{r \gamma}{p} \mu} \lambda^{-r \gamma} \geq m_{\Psi} \mathrm{d} \lambda^{-r \gamma} \equiv D,
$$

for $0<\sigma<\min \left\{\sigma_{0}, \Sigma_{\delta}\right\}$. Note also that for $0<\lambda \leq \lambda_{0}(d)$, then inequality (3.11) entails

$$
\Delta u_{0}+D u_{0}^{p} \geq 2 u_{0}^{p}
$$

for any $0<\delta \leq \delta_{0}$. The comparison principle in conjunction with (3.39) and (3.40) then yields

$$
z \geq \tilde{z} \text { in } Q_{0} \equiv \Omega_{0} \times\left(0, \min \left\{\sigma_{0}, \Sigma_{\delta}\right\}\right),
$$

where $\tilde{z}=\tilde{z}(x, t)$ solves the following partial differential equation

$$
\begin{aligned}
& \tilde{z}_{\sigma}=\Delta \tilde{z}+D \tilde{z}^{p}, \quad \text { in } Q_{0}, \\
& \frac{\partial \tilde{z}}{\partial v}=0, \quad \text { on } \quad \partial \Omega_{0} \times\left(0, \min \left\{\sigma_{0}, \Sigma_{\delta}\right\}\right), \\
& \tilde{z}(|x|, \sigma)=u_{0}(|x|) \text { in } \Omega_{0} .
\end{aligned}
$$

Setting $h(x, \sigma):=\tilde{z}_{\sigma}(x, \sigma)-\tilde{z}^{p}(x, \sigma)$, then

$$
h_{\sigma}=\Delta h+p(p-1) \tilde{z}^{p-2}|\nabla \tilde{z}|^{2}+D p \tilde{z}^{p-1} h \geq \Delta h+D p \tilde{z}^{p-1} h, \quad \text { in } \quad Q_{0},
$$

with

$$
\begin{aligned}
h(x, 0)= & \Delta \tilde{z}(x, 0)+D \tilde{z}^{p}(x, 0) \\
& -\tilde{z}^{p}(x, 0)=\Delta u_{0}+(D-1) u_{0}^{p} \geq u_{0}^{p}>0, \text { in } \Omega_{0},
\end{aligned}
$$

whilst $\frac{\partial h}{\partial v}=0$ on $\partial \Omega_{0} \times\left(0, \min \left\{\sigma_{0}, \Sigma_{\delta}\right\}\right)$. Therefore, owing to the maximum principle, we derive $\tilde{z}_{\sigma}>\tilde{z}^{p}$ in $Q_{0}$, and thus via integration we obtain

$$
\tilde{z}(0, \sigma) \geq\left(\frac{1}{z_{0}^{p-1}(0)}-(p-1) \sigma\right)^{-\frac{1}{p-1}}=\left\{\left(\frac{\delta^{a}}{\lambda\left(1+\frac{a}{2}\right)}\right)^{p-1}-(p-1) \sigma\right\}^{-\frac{1}{p-1}}
$$


for $0<\sigma<\min \left\{\sigma_{0}, \Sigma_{\delta}\right\}$, and therefore,

$$
\min \left\{\sigma_{0}, \Sigma_{\delta}\right\}<\frac{1}{p-1} \cdot\left(\frac{\delta^{a}}{\lambda\left(1+\frac{a}{2}\right)}\right)^{p-1} .
$$

Note that for $0<\delta \ll 1$, the right-hand side on (3.45) is less than $\sigma_{0}$, so $\Sigma_{\delta}<$ $\frac{1}{p-1}\left(\frac{\delta^{a}}{\lambda\left(1+\frac{a}{2}\right)}\right)^{p-1}<+\infty$.

Remark 3.2 Recalling that $z=\mathrm{e}^{\int^{\sigma} \Phi(s) d s} u$, we also obtain the occurrence of a singlepoint blow-up for the solution $u$ of problem (3.3)-(3.5).

Remark 3.3 Notably, by (3.45) we conclude that $\Sigma_{\delta} \rightarrow 0$ as $\delta \rightarrow 0$, i.e. the more spiky initial data we consider then the faster the diffusion-driven blow-up for $z$ and consequently for $u$ as well.

A diffusion-driven instability (Turing instability) phenomenon, as was first indicated in the seminal paper (Turing 1952), is often followed by pattern formation. A similar situation is observed as a consequence of the driven-diffusion finite-time blow-up provided by Theorem 3.1, and it is described below. The blow-up rate of the solution $u$ of (3.3)-(3.5) and the blow-up pattern (profile) identifying the formed pattern are given.

Theorem 3.2 Take $N \geq 3, \max \left\{r, \frac{N}{N-2}\right\}<p<\frac{N+2}{N-2}$ and $\frac{2}{N}<\frac{p-1}{r}<\gamma$. Assume that both $\Phi(\sigma)$ and $\Psi(\sigma)$ are positive and bounded. Then, the blow-up rate of the solution of (3.3)-(3.5) can be characterized as follows

$$
\|u(\cdot, \sigma)\|_{\infty} \approx\left(\Sigma_{\max }-\sigma\right)^{-\frac{1}{p-1},} t \uparrow \Sigma_{\max }
$$

where $\Sigma_{\max }$ stands for the blow-up time.

Proof We first perceive that by virtue of (3.16) and in view of the Hölder's inequality, since $p>r$, the following inequality holds

$$
0<K(\sigma)=\frac{\Psi(\sigma) \mathrm{e}^{(1+r \gamma-p) \int^{\sigma} \Phi(s) \mathrm{d} s}}{\left(f_{\Omega_{0}} z^{r}\right)^{\gamma}} \leq C_{1}<+\infty .
$$

Define now $\Theta$ satisfying the partial differential equation

$$
\Theta_{\sigma}=\Delta \Theta+C_{1} \Theta^{p}, \quad \text { in } \Omega_{0} \times\left(0, \Sigma_{\max }\right),
$$

with $\frac{\partial \Theta}{\partial v}=0$, on $\partial \Omega_{0} \times\left(0, \Sigma_{\max }\right)$, and $\Theta(x, 0)=z_{0}(x)$, in $\Omega_{0}$, then via comparison $z \leq \Theta$ in $\Omega_{0} \times\left(0, \Sigma_{\max }\right)$. Yet it is known, see Quittner and Souplet (2007, Theorem 44.6), that $|\Theta(x, \sigma)| \leq C_{\eta}|x|^{-\frac{2}{p-1}-\eta}$ for $\eta>0$, and thus

$$
|z(x, \sigma)| \leq C_{\eta}|x|^{-\frac{2}{p-1}-\eta}, \quad \text { for } \quad(x, \sigma) \in \Omega_{0} \times\left(0, \Sigma_{\max }\right) .
$$


Following the same steps as in the proof of Kavallaris and Suzuki (2017, Theorem 9.1), we derive

$$
\lim _{\sigma \rightarrow \Sigma_{\max }} K(\sigma)=\omega \in(0,+\infty)
$$

By virtue of (3.49) and applying Quittner and Souplet (2007, Theorem 44.3(ii)), we can find a constant $C_{U}>0$ such that

$$
\|z(\cdot, \sigma)\|_{\infty} \leq C_{U}\left(\Sigma_{\max }-\sigma\right)^{-\frac{1}{(p-1)}}, \quad \text { in }\left(0, \Sigma_{\max }\right)
$$

Setting $N(\sigma):=\|z(\cdot, \sigma)\|_{\infty}=z(0, \sigma)$, then $N(\sigma)$ is differentiable for almost every $\sigma \in\left(0, \Sigma_{\delta}\right)$, in view of Friedman and McLeod (1985), and $\frac{\mathrm{d} N}{\mathrm{~d} \sigma} \leq K(\sigma) N^{p}(\sigma)$. Notably, $K(\sigma) \in C\left(\left[0, \Sigma_{\max }\right)\right)$ and owing to (3.47) it is bounded in any time interval $[0, \sigma], \sigma<\Sigma_{\max }$; then, upon integration we obtain

$$
\|z(\cdot, \sigma)\|_{\infty} \geq C_{L}\left(\Sigma_{\max }-\sigma\right)^{-\frac{1}{(p-1)}}, \quad \text { in } \quad\left(0, \Sigma_{\max }\right)
$$

for some positive constant $C_{L}$.

Recalling that $z(x, \sigma)=\mathrm{e}^{\int^{\sigma} \Phi(s) d s} u(x, \sigma)$ then (3.50) and (3.51) entail

$\widetilde{C}_{L}\left(\Sigma_{\max }-\sigma\right)^{-\frac{1}{(p-1)}} \leq\|u(\cdot, \sigma)\|_{\infty} \leq \widetilde{C}_{U}\left(\Sigma_{\max }-t\right)^{-\frac{1}{(p-1)}}, \quad$ for $\quad \sigma \in\left(0, \Sigma_{\max }\right)$,

where now $\widetilde{C}_{L}$, and $\widetilde{C}_{U}$ depend on $\Sigma_{\max }$, and thus (3.46) is proved.

Remark 3.4 We first note that (3.48) provides a rough form of the blow-up pattern for $z$ and thus for $u$ as well. Additionally, owing to (3.47) the non-local problem (3.12)(3.14) can be treated as a local one for which the more accurate asymptotic blow-up profile, Duong et al. (Duong et al. 2020) and Merle and Zaag (Merle and Zaag 1998), is known and is given by $\lim _{\sigma \rightarrow \Sigma_{\max }} z(|x|, \sigma) \sim C\left[\frac{|\log | x||}{|x|^{2}}\right]$, for $|x| \ll 1$, and $C>0$. Using again the relation between $z$ and $u$, we end up with a similar asymptotic blowup profile for the diffusion-driven-induced blow-up solution $u$ of problem (3.3)-(3.5). This blow-up profile actually determines the form of the developed patterns which are induced as a result of the diffusion-driven instability, and it is numerically investigated in the next section.

\section{Numerical Experiments}

To confirm and illustrate some of the theoretical results of the previous sections, we perform a series of numerical experiments for which we solve the involved PDE problems using the finite element method (Johnson 1987), using piecewise linear basis functions and implemented using the adaptive finite-element toolbox ALBERTA (Schmidt and Siebert 2005). In all our simulations (unless stated otherwise), the domain was triangulated using 16384 elements, the discretisation in time was done using the 
forward Euler method taking $5 \times 10^{-4}$ as time-step and the resulting linear system solved using Generalized Minimal Residual iterative solver (Saad 2003).

\subsection{Experiment 1}

We take an initial condition $u_{0}$ and a set of parameters satisfying the assumptions of Theorem 2.1. Then, solve (1.32)-(1.34) on $\Omega_{0}=[-1,1]^{2}$ with initial condition of the form

$$
u_{0}(\mathbf{x}, 0)= \begin{cases}-8\|\mathbf{x}\|^{2}+3, & \|\mathbf{x}\|<\frac{1}{2} \\ 1, & \text { otherwise }\end{cases}
$$

As for the domain evolution, we consider four different cases:

- $\rho(t)=\mathrm{e}^{\beta t}$ (exponentially growing domain);

- $\rho(t)=\mathrm{e}^{-\beta t}$ (exponentially decaying domain);

- $\rho(t)=\frac{\mathrm{e}^{\beta t}}{1+\frac{1}{m}\left(\mathrm{e}^{\beta t}-1\right)}$ (logistically growing domain);

- $\rho(t)=1$ (static domain).

We summarise all parameters used in Table 1. In Fig. 1, we demonstrate the $\|u(x, t)\|_{\infty}$ for each of the domain evolutions, so we can monitor their respective blow-up times.

If we denote by $\Sigma_{g}, \Sigma_{d}, \Sigma_{l g}$ and $\Sigma_{s}$ the blow-up times for the case of exponentially growing and decaying, the logistically growing domains and the static

Table 1 Set of parameters used in Experiment 1

\begin{tabular}{lllllll}
\hline$D_{1}$ & $p$ & $q$ & $r$ & $s$ & $\beta$ & $m$ \\
\hline 1 & 3 & 2 & 1 & 2 & 0.1 & 1.5 \\
\hline
\end{tabular}

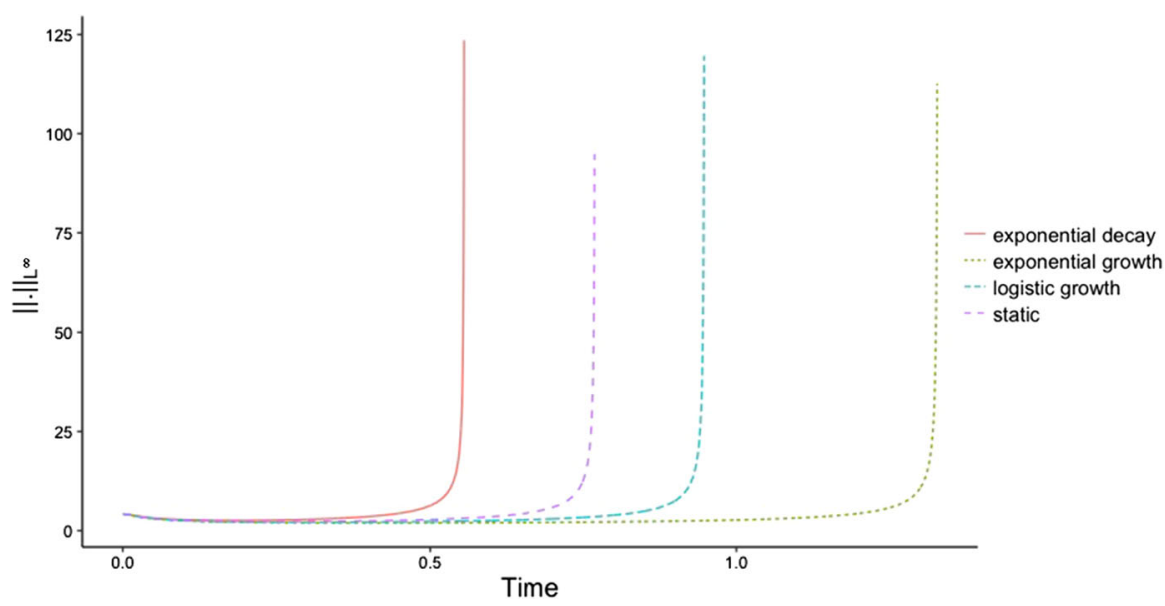

Fig. 1 Plots representing $\|u(x, t)\|_{\infty}$, where $u(x, t)$ is the numerical solution of (1.32)-(1.34) for different domain evolutions: static, exponentially decaying and growing, and logistically growing domains, starting from the initial condition (4.1) in $\Omega_{0}=[-1,1]^{2}$. Parameters are shown in Table 1 and satisfy conditions of Theorem 2.1. (Colour version online) 


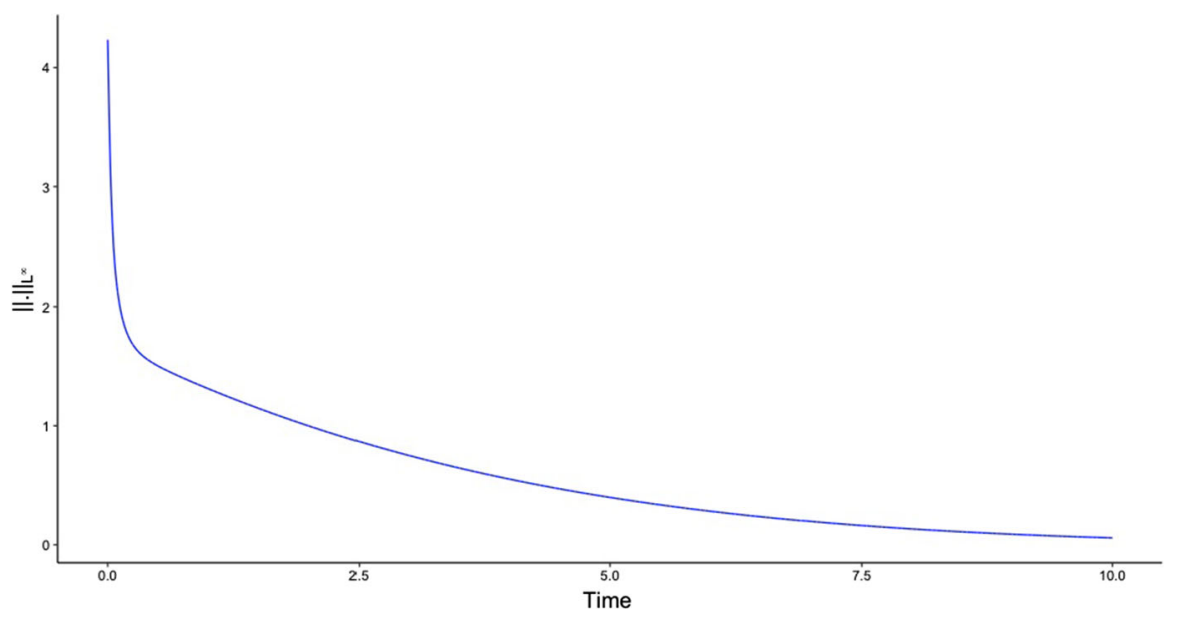

Fig. 2 The plot of $\|\bar{u}\|_{\infty}$ resulting from the numerical solution of (1.32)-(1.34) starting from the initial condition (4.1) in $\Omega_{0}=[-1,1]^{2}$, evolving exponentially, considering parameters $D_{1}=1, \beta=0.1$, $p=1.4, q=1, r=1$ and $s=2$. (Colour version online)

domain, respectively, we observe from Fig. 1 that we have the following ordering $\Sigma_{g}>\Sigma_{l g}>\Sigma_{s}>\Sigma_{d}$, which is in agreement with the mathematical intuition, but it cannot be derived by our analytical results cf. Remark 2.4.

We now take the same initial condition, $u_{0}$ and the same initial domain which we assume is evolving exponentially and consider parameters $D_{1}=1, p=1.4$, $q=1, r=1$ and $s=2$ for which inequality (2.24) of Remark 2.6 holds. As we can see in Fig. 2, we have an example of a solution $u$ for which its mean value $\bar{u}$ does not blow up, as already conjectured in the aforementioned remark. Hence, this numerical experiment predicts a very interesting phenomenon both mathematically and biologically which has been conjectured but not proven by Theorem 2.1. It predicts the infinite-time quenching of the solution of problem (1.32)-(1.34), and thus, the extinction of the activator in the long run, see also Remark 2.3. It must also be noted that this result is not in contradiction with Proposition 2.2, where infinite-time quenching is ruled out since condition (2.1) is not satisfied for an exponentially growing domain where $\Phi(\sigma)$ is an unbounded function as indicated in Remark 2.4.

\subsection{Experiment 2}

This experiment is meant to illustrate Theorem 2.3, and we take as initial data $u_{0}=$ $\cos (\pi y)+2$ and take $\Omega_{0}$ as the unit square when numerically solving equations (1.32)(1.34). As for domain evolution, we consider $\rho(t)=\mathrm{e}^{\beta t}$, with $\beta=0.1$. To proceed, we consider two sets of parameters, one for which assumptions of Theorem 2.3 are satisfied and another for which those assumptions are not fulfilled. See Table 2 for model parameters. 
Table 2 Set of parameters used in Experiment 2

\begin{tabular}{llllll}
\hline Conditions of Theorem 2.3 & $D_{1}$ & $p$ & $q$ & $r$ & $s$ \\
\hline Are verified & 1 & 1 & 2 & 3 & 2 \\
Are not verified & 1 & 3 & 2 & 1 & 1 \\
\hline
\end{tabular}

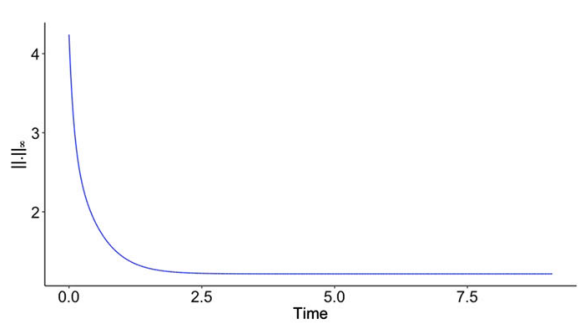

(a) Conditions of Th. 2.3 are met $(p=1, q=2, r=3, s=2)$.

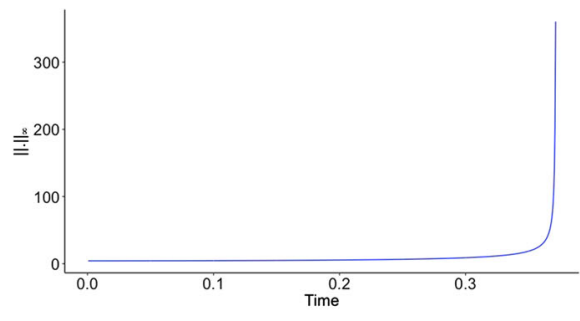

(b) Conditions of Th. 2.3 are not met $(p=3, q=2, r=1, s=2)$.

Fig. 3 The plot of $\|u(x, t)\|_{\infty}$, where $u(x, t)$ is the numerical solution of (1.32)-(1.34). Initial condition is $u_{0}=\cos (\pi y)+2$, and $\Omega_{0}$ is the unit square evolving according to exponential growth $(\beta=0.1)$. (Colour version online)

Results shown in Fig. 3 are in agreement with theoretical predictions of Theorem 2.3 since the solutions exist for all times when the assumption of the theorem is met (Fig. 3a), otherwise, a finite-time blow-up is exhibited to occur (Fig. 3b).

\subsection{Experiment 3}

In this experiment, we intend to illustrate Theorem 3.1, so we numerically solve (1.32)(1.34) in $\mathbb{R}^{3}$, taking $\Omega_{0}$ as the unit sphere and initial condition $u_{0}$ given by (3.1), considering $\delta=0.8$ and $\lambda=0.1$. As for other parameters, we choose $D_{1}=1, p=4$, $q=4, r=2$ and $s=1$, which satisfy the conditions of the theorem. In Fig. 4, we display the $L^{\infty}$-norm of the solution $u$ for three types of evolution laws implemented, namely: exponential decay, logistic decay and no evolution. For the exponential and logistic decay, we select the same set of parameters as used in Experiment 1. As we can observe, for all the cases the solution blows up, as theoretically predicted by Theorem 3.1. Again the blow-up times satisfy the ordering $\Sigma_{s}>\Sigma_{l s}>\Sigma_{d}$, where $\Sigma_{l s}$ stands for the blow-up time for the logistic decay evolution, being in agreement with the mathematical intuition. Such an ordering, again, cannot be obtained via the theoretical result of Theorem 3.1.

In Fig. 5a, b, we compare the initial solution with the solution at $t=0.03$, respectively, for the logistic decay, close to the blow-up time $t=0.03$, by looking at a cross section of the three-dimensional unit sphere $\Omega_{0}$. Besides, in Fig. 5c, d again the solution at section cross of $\Omega_{0}$ is depicted but now for the stationary and exponential decaying case, respectively. Through this experiment, we can observe the formation of blow-up (Turing-instability) patterns around the origin $R=0$. We actually conclude that the evolution of the domain has no impact on the form of blow-up patterns; 
Fig. 4 Plots for $\|u(x, t)\|_{\infty}$, where $u(x, t)$ is the numerical solution of (1.32)-(1.34), in $\mathbb{R}^{3}$, considering $\Omega_{0}$ the unit sphere.

Three evolution laws considered: exponential decay, logistic decay and no evolution (static domain). Parameters used: $p=4, q=4$, $r=2, s=1$ and initial condition given by (3.1) taking $\delta=0.8$ and $\lambda=0.1$. (Colour version online)

(a)

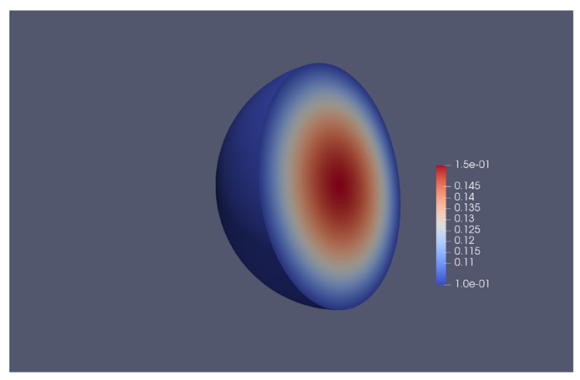

(c)

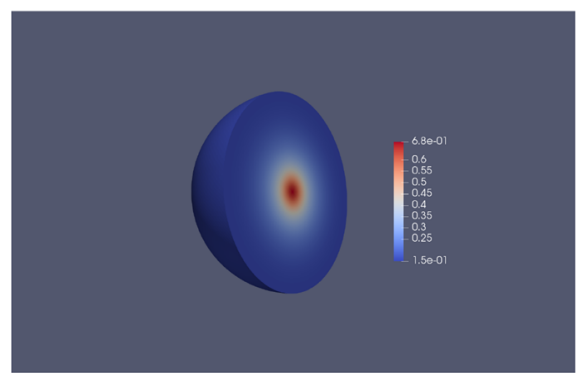

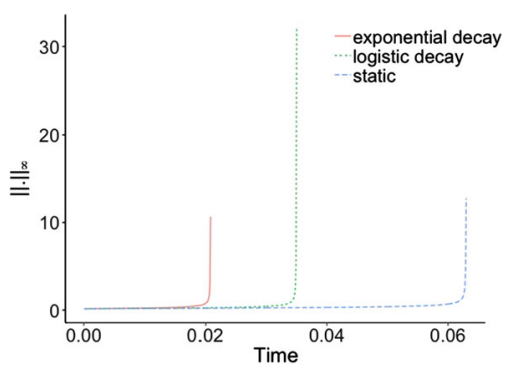

(b)

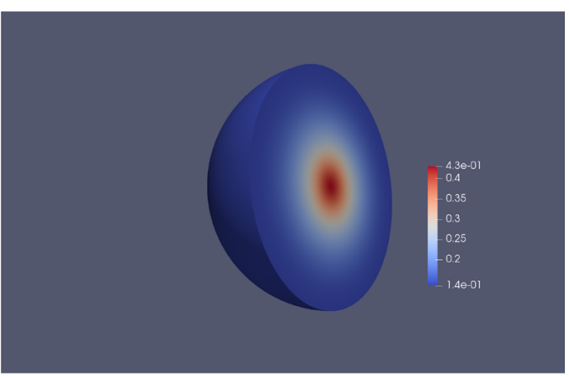

(d)

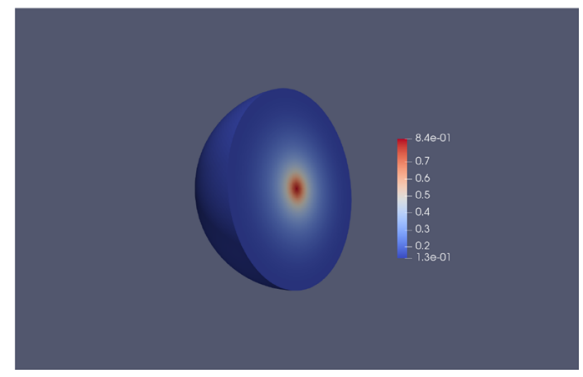

Fig. 5 Numerical solution of experiment 3. The above figures show the blow-up (Turing-instability) patterns on a cross section of the three-dimensional sphere $\Omega_{0}$ : a initial profile of solution for logistic growth; $\mathbf{b}$ blow-up pattern for logistic growth at $t=0.03$; c blow-up pattern for stationary case at $t=0.07$; and, $\mathbf{d}$ blow-up pattern for exponential decay at $t=0.03$.(Colour version online)

however, it certainly affects the spreading of Turing-instability patterns as it is obvious from Fig. 5b, c, d.

Notably Theorem 3.1 holds only to $N \geq 3$; however, we have numerically tested the occurrence of blow-up predicted by that theorem also for $N=2$, taking $\Omega$ as the unit circle and the same parameters used in Experiment 3. It is then numerically verified the exhibition of finite-time blow-up. The numerical results are displayed, in Fig. 6 where the $L^{\infty}$-norm of the solution $u$ for the same three types of evolution laws is depicted for the $N=3$ case. The initial condition used is displayed in Fig. 7. 
Fig. 6 Plots for $\|u(x, t)\|_{\infty}$, where $u(x, t)$ is the numerical solution of (1.32)-(1.34), in $\mathbb{R}^{2}$, considering $\Omega_{0}$ the unit circle. Three domain evolution laws considered: exponential decay, logistic decay and no evolution (static domain). Parameters used: $p=4, q=4, r=2$, $s=1$ and initial condition given by (3.1) taking $\delta=0.8$ and $\lambda=0.1$. (Colour version online)

Fig. 7 Initial condition used for Experiment 3 when $N=2, \Omega_{0}$ is the unit circle and initial condition is given by (3.1) taking $\delta=0.8$ and $\lambda=0.1$. (Colour version online)
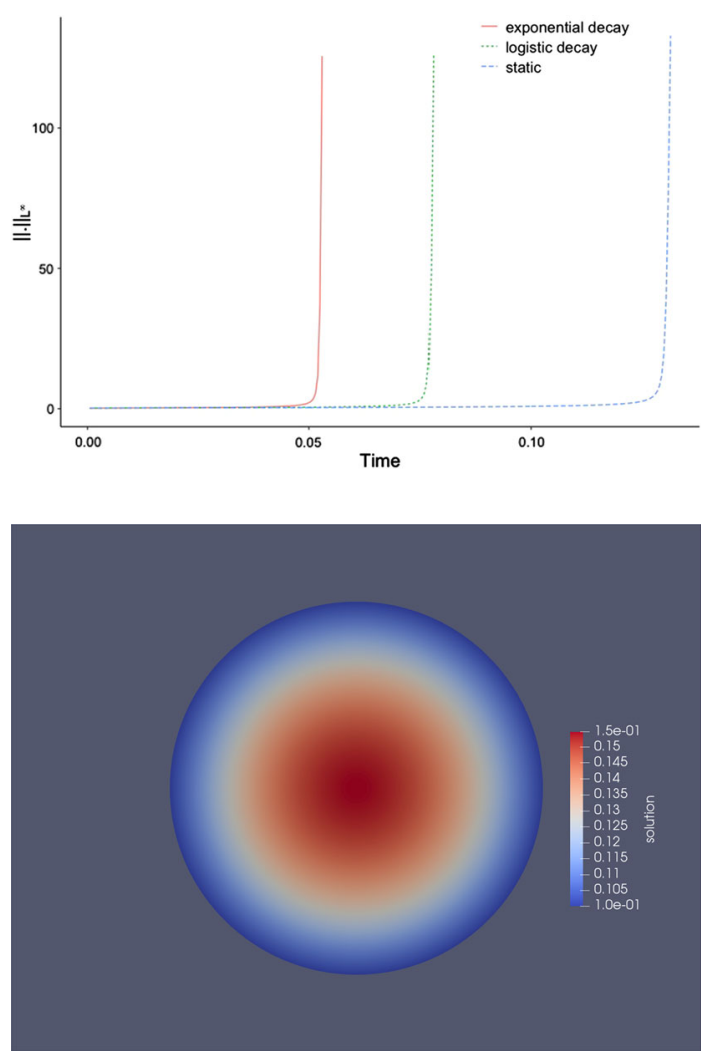

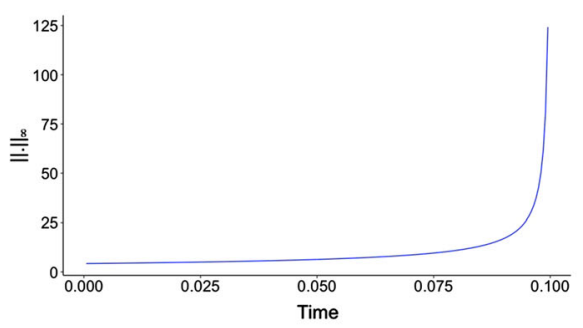

(a) $\|\hat{u}\|_{\infty}$ for the numerical solution of (1.18)-(1.19).

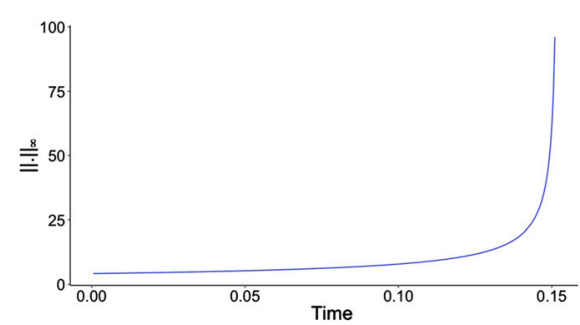

(b) $\|u\|_{\infty}$ for the numerical solution of (1.32)-(1.34).

Fig. 8 Plots of the $L_{\infty}$ norm for the numerical solutions of (1.18)-(1.19) and (1.32)-(1.34). The initial condition is prescribed as $u_{0}=\hat{u}_{0}=\cos (\pi y)+2$ where $\Omega_{0}$ is an exponentially decaying unit square with $\beta=0.1$. (Colour version online)

\subsection{Experiment 4}

Next, we design a numerical experiment to compare the dynamics of the reactiondiffusion system (1.18)-(1.19) with that of the non-local problem (1.32)-(1.34) under the assumptions of Theorem 2.1. To this end, we perform an experiment considering 
$u_{0}=\hat{u}_{0}=\cos (\pi y)+2, \Omega_{0}=[0,1]^{2}, p=3, q=2, r=1$ and $s=2$. For the reaction-diffusion system (1.18)-(1.19), we also take in addition $D_{1}=0.01, D_{2}=1$, $\tau=0.01$ and $v_{0}=2$ whilst for (1.32)-(1.34) we only choose $D_{1}=0.01$. For both cases, we consider an exponential decaying evolution of the domain, with $\beta=0.1$. Unlike previous numerical examples, here the domain was triangulated using 786432 elements and a timestep $10^{-4}$ was taken.

The obtained results are displayed in Fig. 8, and they demonstrate that the reactiondiffusion system (1.18)-(1.19), and the non-local problem (1.32)-(1.34) share the same long time dynamics. In particular, the solutions of both problems exhibit blowup which takes place in finite time. The latter, biologically speaking, means that in the examined case we just need to monitor only the dynamics of the activator, whose dynamics are governed by the non-local problem (1.32)-(1.34). Therefore, we can get an insight regarding the interaction between both of the chemical reactants (activator and inhibitor) provided by the reaction-diffusion system (1.18)-(1.19).

Acknowledgements RB acknowledges support from the National Funding from FCT_-Fundação para a Ciência e a Tecnologia, under the Project: UID/MAT/04561/2019. This work (AM) is partly supported by the EPSRC Grant Number EP/J016780/1, the European Union Horizon 2020 research and innovation programme under the Marie Sklodowska-Curie Grant Agreement No. 642866, the Commission for Developing Countries, and the Simons Foundation. AM is a Royal Society Wolfson Research Merit Award Holder funded generously by the Wolfson Foundation. AM is also a Distinguished Visiting Scholar to the University of Johannesburg, Department of Mathematics, South Africa. AM is a Distinguished Visiting Scholar to the Universita degli Studi di Bari Aldo Moro, Bari, Italy.

Open Access This article is licensed under a Creative Commons Attribution 4.0 International License, which permits use, sharing, adaptation, distribution and reproduction in any medium or format, as long as you give appropriate credit to the original author(s) and the source, provide a link to the Creative Commons licence, and indicate if changes were made. The images or other third party material in this article are included in the article's Creative Commons licence, unless indicated otherwise in a credit line to the material. If material is not included in the article's Creative Commons licence and your intended use is not permitted by statutory regulation or exceeds the permitted use, you will need to obtain permission directly from the copyright holder. To view a copy of this licence, visit http://creativecommons.org/licenses/by/4.0/.

\section{References}

Bobrowski, A., Kunze, M.: Irregular convergence of mild solutions of semilinear equations. J. Math. Anal. Appl. 472, 1401-1419 (2019)

Brezis, H.: Functional Analysis, Sobolev Spaces and Partial Differential Equations. Springer, New York (2011)

Cusseddu, D., Edelstein-Keshet, L., Mackenzie, J.A., Portet, S., Madzvamuse, A.: A coupled bulk-surface model for cell polarisation. J. Theor. Biol. 48(21), 119-135 (2019)

Duong, G.K., Kavallaris, N.I., Zaag, H.: Diffusion-induced blowup solutions for the shadow limit model of a singular Gierer-Meinhardt system, preprint (2020)

Evans, L.C.: Partial Differential Equations, 2nd edn. Graduate Studies in Mathematics, vol. 19. AMS, Providence (2010)

Fila, M., Ninomiya, H.: Reaction versus diffusion: blow-up induced and inhibited by diffusivity. Russ. Math. Surv. 60(6), 1217-1235 (2005)

Friedman, A., McLeod, J.B.: Blow-up of positive solutions of semilinear heat equations. Indiana Univ. Math. J. 34, 425-447 (1985)

Gierer, A., Meinhardt, H.: A theory of biological pattern formation. Kybernetik (Berlin) 12, 30-39 (1972)

Hu, B., Yin, H.-M.: Semilinear parabolic equations with prescribed energy. Rend. Circ. Mat. Palermo 44, 479-505 (1995) 
Jiang, H.: Global existence of solutions of an activator-inhibitor system. Discrete Contin. Dyn. Syst. 14, 737-751 (2006)

Johnson, C.: Numerical Solution of Partial Differential Equations by the Finite Element Method. Cambridge University Press, Cambridge (1987)

Karali, G., Suzuki, T., Yamada, Y.: Global-in-time behavior of the solution to a Gierer-Meinhardt system. Discrete Contin. Dyn. Syst. 33, 2885-2900 (2013)

Karch, G., Suzuki, K., Zienkiewicz, J.: Finite-time blowup of solutions to some activator-inhibitor systems. Discrete Contin. Dyn. Syst. 36(9), 4997-5010 (2016)

Kavallaris, N.I., Suzuki, T.: On the dynamics of a non-local parabolic equation arising from the GiererMeinhardt system. Nonlinearity 30, 1734-1761 (2017)

Kavallaris, N.I., Suzuki, T.: Non-Local Partial Differential Equations for Engineering and Biology: Mathematical Modeling and Analysis, Mathematics for Industry, vol. 31. Springer, New York (2018)

Keener, J.: Activators and inhibitors in pattern formation. Stud. Appl. Math. 59, 1-23 (1978)

Kondo, S., Asai, R.: A reaction-diffusion wave on the skin of the marine angelfish Pomacanthus. Nature 376, 765-768 (1995)

Labadie, M.: The stabilizing effect of growth on pattern formation, preprint (2008)

Lieberman, G.M.: Second Order Parabolic Differential Equations. World Scientific Publishing Co., Inc., River Edge (1996)

Li, M., Chen, S., Qin, Y.: Boundedness and blow up for the general activator-inhibitor model. Acta Math. Appl. Sin. 11, 59-68 (1995)

Li, F., Ni, W.-M.: On the global existence and finite time blow-up of shadow systems. J. Differ. Equ. 247, 1762-1776 (2009)

Li, F., Peng, R., Song, X.: Global existence and finite time blow-up of solutions of a Gierer-Meinhardt system. J. Diff. Equ. 262(1), 559-589 (2017)

Li, F., Yip, N.K.: Finite time blow-up of parabolic systems with nonlocal terms. Indiana Univ. Math. J. 63(3), 783-829 (2014)

Madzvamuse, A., Maini, P.K.: Velocity-induced numerical solutions of reaction-diffusion systems on continuously growing domains. J. Comput. Phys. 225, 100-119 (2007)

Masuda, K., Takahashi, K.: Reaction-diffusion systems in the Gierer-Meinhardt theory of biological pattern formation. Jpn. J. Appl. Math. 4, 47-58 (1987)

Merle, F., Zaag, H.: Refined uniform estimates at blow-up and applications for nonlinear heat equations. Geom. Funct. Anal. 8(6), 1043-1085 (1998)

Mizoguchi, N., Ninomiya, H., Yanagida, E.: Diffusion-induced blowup in a nonlinear parabolic system. J. Dyn. Differ. Equ. 10(4), 619-638 (1998)

Ni, W.-M.: The Mathematics of Diffusion CBMS-NSF Series. SIAM, Philadelphia (2011)

Ni, W.-M., Suzuki, K., Takagi, I.: The dynamics of a kinetic activator-inhibitor system. J. Differ. Equ. 229, 426-465 (2006)

Plaza, R.G., Sanchez-Garduno, F., Padilla, P., Barrio, R.A., Maini, P.K.: The effect of growth and curvature on pattern formation. J. Dyn. Differ. Equ. 16(4), 1093-1121 (2004)

Quittner, P., Souplet, p: Superlinear Parabolic Problems, Blow-up, Global Existence and Steady States. Birkhäuser, Basel (2007)

Rothe, F.: Global Solutions of Reaction-Diffusion Equations, Lecture Notes in Mathematics, vol. 1072. Springer, Berlin-Heidelberg-New York (1984)

Saad, Y.: Iterative Methods for Sparse Linear Systems, 2nd edn. SIAM, Philadelphia (2003)

Schmidt, A., Siebert, K.G.: Design of Adaptive Finite Element Software: The Finite Element Toolbox ALBERTA. Springer, Newe York (2005)

Turing, A.M.: The chemical basis of morphogenesis. Phil. Trans. R. Soc. B 237, 37-72 (1952)

Wei, J.: Existence and Stability of Spikes for the Gierer-Meinhardt System, Handbook of Differential Equations: Stationary Partial Differential Equations, vol. 5, pp. 487-585. Elsevier/North-Holland, Amsterdam (2008)

Zou, H.: Finite-time blow-up and blow-up rates for the Gierer-Meinhardt system. Appl. Anal. 94(10), 2110-2132 (2015)

Publisher's Note Springer Nature remains neutral with regard to jurisdictional claims in published maps and institutional affiliations. 


\section{Affiliations}

\section{Nikos I. Kavallaris ${ }^{1}\left[\right.$. Raquel Barreira ${ }^{2,3} \cdot$ Anotida Madzvamuse $^{4,5,6}$}

$\bowtie \quad$ Nikos I. Kavallaris

n.kavallaris@chester.ac.uk

Raquel Barreira

raquel.barreira@estbarreiro.ips.pt

Anotida Madzvamuse

a.madzvamuse@sussx.ac.uk

https://profiles.sussex.ac.uk/p136962-anotida-madzvamuse

1 Department of Mathematical and Physical Sciences, University of Chester, Thornton Science Park Pool Lane, Ince, Chester CH2 4NU, UK

2 Barreiro School of Technology of the Polytechnic Institute of Setubal, Rua Americo da Silva Marinho-Lavradio, 2839-001 Barreiro, Portugal

3 Center of Mathematics, Fundamental Applications and Operations Research (CMAFcIO), University of Lisbon, Lisbon, Portugal

4 School of Mathematical and Physical Sciences, Department of Mathematics, University of Sussex, Falmer, Brighton BN1 9QH, England, UK

5 Department of Mathematics, University of Johannesburg, Johannesburg, South Africa

6 Universita degli Studi di Bari Aldo Moro, Bari, Italy 